Sharif University of Technology
Scientia Iranica
Transactions B: Mechanical Engineering
hCIENTIA

Research Note

\title{
Automated CFD-based optimization of inverted bow shape of a trimaran ship: An applicable and efficient optimization platform
}

\author{
A. Nazemian and P. Ghadimi* \\ Department of Maritime Engineering, Amirkabir University of Technology, Tehran, P.O. Box 15875-4413, Iran.
}

Received 25 August 2020; received in revised form 4 November 2020; accepted 7 December 2020

\author{
KEYWORDS \\ Trimaran hull; \\ Arbitrary Shape \\ Deformation (ASD); \\ CFD; \\ Optimization; \\ Total resistance; \\ Surrogated model.
}

\begin{abstract}
This paper investigates the improvement of the bow region of the trimaran ship hull, and proposes a Computational Fluid Dynamics (CFD)-based automated method to reduce total resistance. The main objectives pursued in the present study include: 1) to create and develop a useful optimization platform to modify the ship hull and 2 ) to investigate the effect of different inverted bow on the hydrodynamic performance of trimaran ship. A wave-piercing bow trimaran hull was taken as the baseline design. The ship bow has been redesigned using Arbitrary Shape Deformation (ASD) technique, which defines the input variables for the optimization process. The objective function was the drag force, and this study is conducted at cruise speed. To accomplish this task, two optimization methods were sequentially applied. A Latin Hypercube Sampling tool distributes design points and an Radial Basis Function (RBF)-based surrogate model is constructed to investigate system behavior. The final optimum design of Design Of Experiment (DOE) study was introduced to the direct optimization SHERPA algorithm as a baseline design. The integration of CFD solver, geometric parameterization and optimizer tools is managed by the HEEDS MDO software package using a multi-connection method. The optimization results show that the optimization was successfully carried out and the resistance was reduced by $10.2 \%$. The comparison between the initial hull and the optimized hull shows that the proposed optimization platform can be used for ship hull optimization in industrial application and significantly reduces the computational time and workload.
\end{abstract}

(C) 2021 Sharif University of Technology. All rights reserved.

\section{Introduction}

To reduce resistance and design effective structures, improving the hydrodynamic performance of marine vehicles has attracted widespread attention from re-

\footnotetext{
*. Corresponding author. Tel.: +982164543110; Fax: +982166412495

E-mail address: pghadimi@aut.ac.ir (P. Ghadimi)
}

searchers. An optimized hull form that does not reduce buoyancy has been developed, and the result of this evolution is the introduction of a new hydrodynamic optimization process. The marine vehicle optimization process is related to the flow around the vehicle, especially the bow region that has an important role in hull resistance. Hence, this work introduces a design optimization solution for reducing the total resistance (drag). This platform which is based on the Computational Fluid Dynamics (CFD) simulation technique was applied to the hull of the wave 
piercing bow trimaran. The optimization process was carried out on a new and complex inverted bow shape trimaran hull, for which there was no sufficient prior knowledge and information. In the past few years, this has led to extensive research on the analysis of flow characteristics [1]. Regarding the resistance and its components, extensive research has been conducted on the trimaran design. However, limited research has been devoted to hullform optimization, especially for novel and complex multihull ships. Most of the research has been devoted to the sidehull arrangement, the main part of which was focused on the resistance analysis, and the research on the seakeeping performance is very limited. Wilson et al. [2] proposed the idea of wave-canceling around trimaran hullform based on different sidehull configurations. This phenomenon leads to constructive interaction, which reduces the resistance to wave-making. In addition, Suzuki and Ikehata [3] and Brizzollara et al. [4] also conducted numerical and experimental studies on the resistance of the trimaran vessels and adopted practical methods to reduce it. Ghadimi et al. [5] investigated the effect of sidehull arrangement on seakeeping of trimaran. The results showed that proper sidehull transversal and longitudinal distances can improve the seakeeping performance of the trimaran. However, most of the research has been conducted without an automated cycle of the design study. Jia et al. [6] studied the resistance and seakeeping characteristics of the transom stern trimaran. They conducted parametric investigations on different Froude numbers and sidehull arrangements.

Wang et al. conducted another comprehensive study on the seakeeping of trimaran [7]. They investigated the optimum position of trimaran outriggers by using Non-dominated Sorting Genetic Algorithm II (NSGA-II) optimization algorithm and potential-based $2.5 \mathrm{D}$ solver. Their method was very effective, but the optimization space was limited to the sidehull arrangement. By combining different disciplines, Zong et al. [8] developed a CFD-based hull optimization method for a trimaran. The ship hull was modified by the selfblending method and was combined with a CFD solver and MIGA optimizer algorithm. In addition, they also studied the position of the sidehull arrangement relative to the main hull. There are extensive studies on the general characteristics of trimaran ships and sidehull configurations $[9,10]$. However, there is still a lack of a suitable and effective optimization platform to apply CFD-based shape optimization to the hulls of a trimaran; especially it is true of the modification of the inverted bow shape. Consequently, a Simulation-Based Design (SBD) study was conducted on a wave-piercing trimaran hull bow. Using search-based optimization algorithm requires an automatic optimization cycle to control the design variables to reach the final optimum values. Hence, the automated optimization approach is the first achievement of the present paper. Therefore, the use of the heuristic novel SHERPA algorithm in the automation platform can produce successful optimization without user intervention.

The automated optimization platform involves different disciplines, such as Computer-Aided Design/ Computer-Aided Manufacturing (CAD/CAM), CFD, and optimization algorithm [11,12]. The CFD solver predicts calm water resistance more accurately than other analytical or potential energy-based resistance calculation methods [13-15]. Hence, in the current work, the CFD solver simulated the fluid flow characteristics around the trimaran hull, thereby calculating the total resistance and its components, and obtaining reliable and accurate results became possible. Another important strategy for numerical ship optimization is geometric reconstruction. During the optimization process, the design variables will be adjusted according to the optimization algorithm, and then the design variables will be turned back to change the hull shape. To obtain the best performance of the optimized platform, some aspects need to be considered, such as smoothness, geometric expressions with a small number of variables, and a wide design space $[1,16,17]$. Researchers develop different applicants based on platform integrators. Tahara et al. [18] combined CADbased NAPA software with a CFD solver to optimize a container ship. Chen and Chen [19] developed a nonlinear potential flow solver and optimized the hullform using the CAD environment. One of the comprehensive studies of the geometry modification approach was applied by Brizzolara et al. [20] who compared the full parametric hullform definition and the Free Form Deformation (FFD) technique. A complete trial of ship parameterization has been carried out again by Harries et al. [21], Abt [22], and Harries [23]. They introduced different types of coupling between CFD and CAD. Han et al. [24] studied how to develop a parametric geometry to describe the hydrodynamic optimization of hull forms. Vasudev et al. [25] studied ship hull optimization by using a multi-objective optimization platform. Two integrated SHIPFLOW software and the NSGA-II method were used for geometric optimization. Kim et al. [26] established a connection between CAD software friendship and SHIPFLOW software and investigated the optimization of ship form. Kim and Yang [27] and Huang and Yang [28] investigated ship form optimization based on ship geometric parameters and Radial Basis Function (RBF) morph technique. The global modification was performed by shifting method and local variation of ship form was performed by RBF control point displacement.

Recently, Zhang et al. [13] optimized the hull form of the Wigley hull and DTMB5512 model by using the Latin Hypercube Sampling (LHS) method and ap- 
proximation method. The parametric method was the Arbitrary Shape Deformation (ASD) technique, which is also used in this paper. Diez et al. [29] studied the different hydrodynamic problems of the fast catamaran hull. They used stochastic optimization methods to improve the resistance and operability with geometrical and displacement constraints. The optimization tools consisted of Karhunen-Loève expansion of a free-form deformation, URANS-based CFD simulations, metamodels, and multi-objective particle swarm. Another similar attempt was made by Serani et al. [30] that developed a high-fidelity stochastic shape optimization problem. They modified a DTMB5415 model under calm water and wavy conditions by combining stochastic shape optimization and design-space evaluation methods. Guo et al. [31] conducted a comprehensive optimization study of a waterjet-propelled trimaran by using the surrogate model and a direct NSGA-II optimization algorithm. They aimed to obtain the total resistance, thrust and torque, and power consumption of the propulsion system which led to true optimization. Zakerdoost and Ghassemi [32] studied the interaction and optimization of another hull propulsion system. However, the driving force of the above research was single propeller and double propeller. Michell integral and lifting line theory was employed for optimization of the hull-propeller interaction system. Coppedè et al. [33] investigated a CAD-based optimization tool for the improvement of calm water performance of the KRISO Container Ship (KCS) model. They used the FFD method for geometric parametrization and the CFD solver for resistance calculation. Furthermore, a Gaussian Process-Response Surface Method (GP-RSM) based on an ordinary Kriging model has been established to speed up the evaluation of the objective function. Feng et al. [34] applied a CADbased parametric approach to optimize a supply vessel hullform. Their research was conducted on a multiobjective optimization problem due to the modification of the hullform at different operating speeds.

The results following from the review of the field literature lead us to conclude that the hull optimization problem has not been applied to the hull of the wavepiercing bow trimaran. In addition, unlike the previous optimization research, in the present study, a hybrid method was used. The latter was the result of the combination of the Latin hypercube and SHERPA algorithm to solve the hydrodynamic optimization problem. An optimization platform connects different software based on the efficient performance of each discipline. The main purpose of this research was to create and develop a multi-disciplinary optimization platform through the CFD simulation method, which can be used for shape optimization of future fluidexposed geometric problems.

The design spiral of the ship hullform includes different aspects, and it takes a very long time to design and optimize the ship hull. Therefore, an appropriate optimization platform should be fast, flexible, and developable so that optimization can be successfully performed. The third part of the optimization cycle is the optimization algorithm. The two basic characteristics that need to be considered when choosing the best algorithm are ease of implementation and the ability to explore globally. In recent years, many different optimization algorithms have been adopted for hydrodynamic problems [1,35-37]. Optimization methods can be divided into two categories: Gradientfree methods and gradient-based methods. In the Gradient-free methods like Particle Swarm Optimization (PSO) and Genetic Algorithm (GA), amongst other algorithms, only the values of the objective function and the constraint function are required. The gradient-free algorithms explore all parts of the design space. Compared with the gradient-based algorithm, it is very possible to find the global minimum [1]. Besides, the ship optimization problem also involves complex design research that requires a global search.

The present paper offers an automated and lowcost method for improving the hull form of a complex and novel trimaran, which could be extended to other maritime projects. The accomplishment of this task with appropriate calculation time, which is very important in the marine industry, could be ensured through proposed optimization platform and combination of optimization methods. For example, in the absence of a proposed plan, the optimization process takes more than a year of running time and requires a lot of calculation work. In contrast, the proposed optimization platform reduces this time to about 48 days. In terms of time and cost, this is a considerable saving. Therefore, a specific optimization process has been introduced, which provides a convenient and direct way for marine engineers to use flexible tools to solve their optimization problems.

Based on the discussed scenario, the bow region of the wave-piercing bow trimaran was optimized based on the reduction in resistance. To accomplish this task, an ASD technique is firstly applied for CAD parametrization. Second, the initial CFD simulation was computed at ship cruise speed. Later, an optimization cycle was employed by combining approximation and direct solution optimization techniques. The defined objective function is drag force and ASD hull control points are variables, which must be introduced into the CFD solver. The mentioned optimization framework determines these control points to achieve the improvement of the objective function. Finally, the results of hull form optimization are presented and discussed. The initial and optimized hulls are compared, and the optimization framework and its effectiveness are verified. 
Table 1. Specifications of the trimaran ship hull: The main vessel and the model type [37-39].

\begin{tabular}{lcc}
\hline \multicolumn{1}{c}{ Specifications } & Main vessel & Model \\
\hline Overall length (m) & 124 & 3.1 \\
Draft length (m) & 123.2 & 3.08 \\
Total width (m) & 22.2 & 0.555 \\
Main hull width (m) & 5 & 0.125 \\
Side hull length (m) & 36 & 0.9 \\
Draft (m) & 4.384 & 0.1096 \\
Clearance (side hull transverse distance) (m) & 9.7 & 0.2425 \\
Stagger (side hull longitudinal distance) (m) & 0 & 0 \\
Displacement & 2248.8 (Tonnes) & $35(\mathrm{~kg})$ \\
\hline
\end{tabular}

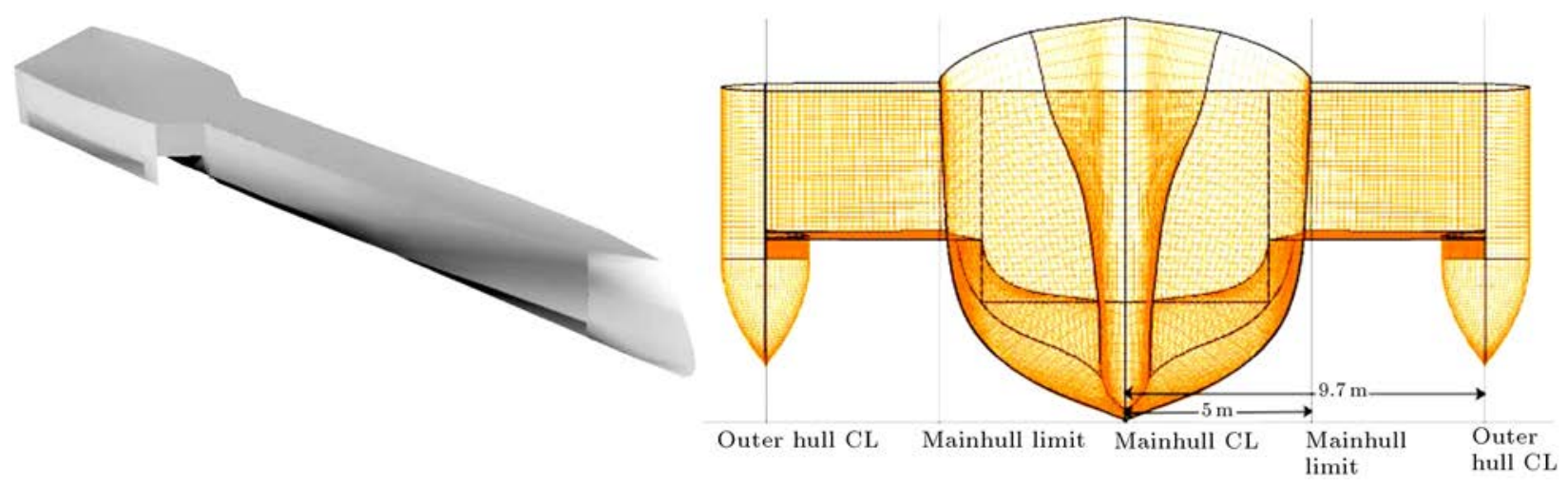

Figure 1. 3D view of the studied trimaran ship.

\section{Problem definition}

The goal of the present paper is to apply an optimization process to optimize the hull form of a trimaran for drag reduction. The present trimaran hull form optimization problem can be defined as follows:

$$
\begin{array}{ll}
\text { minimize } & f(X), \\
\text { subject to } & X \in S \subseteq R^{N},
\end{array}
$$

where $f$ is the objective function that is total resistance and denoted by $R_{T}$. Also, $S \subseteq R^{N}$ is a feasible solution set, while the constraint prohibits feasible design space. A vector of design variables defined by $X$ in $N$ dimension and associated with geometric reconstruction in the optimization process is plotted. Through the displacement constraint of less than $1 \%$, the shape modification and change of the ship hull geometry were realized.

$$
\left|\frac{\Delta_{\text {new }}-\Delta_{\text {org }}}{\Delta_{\text {org }}}\right| \leq 0.01
$$

For trimaran ships, the middle body was considered a slenderer with a longer length to generate the lowest wave-making resistance. Indeed, for this type of ship, resistance can be an advantage when the main body hullform is properly designed. In this study, a model of wave-piercing bow trimaran ships was studied. The dimensional characteristics of this ship and the model $(\lambda=40)$ are shown in Table 1 and the threedimensional view of the trimaran is shown in Figure 1. The ship bow is in the form of a wave-piercing, and the lateral bodies were made in the form of a Wigley hull that has been experimented by Akbari et al. [37].

\subsection{Optimization framework}

This section introduces the integrated part of the optimization framework. The overall structure of the optimization framework includes geometric parameterization, numerical simulation set up, and optimization algorithm. In the current study, ASD technique was used for surface modification to define the local variation of the hull surface during the optimization process. The purpose of this study was to optimize the shape of the bow region of the inverted-bow shape of the multihull ship by minimizing its total drag. A RANSEbased CFD solver was conducted to calculate total resistance. Numerical simulation was performed by STAR CCM+ software and two optimization methods were connected to simulations via HEEDS software. An LHS distributes design samples based on the efficient response, and an RBF interpolation estimates the response of the system. It helps to evaluate the best design based on the minimum resistance. The 
Table 2. Summary of the design variables.

\begin{tabular}{cccll}
\hline No. & Parameters & Control point deformation & Movement & min $\leq$ main $\leq \max (\mathbf{m})$ \\
\hline 1 & $D x 1$ & $t u 6 / s t 1$, st 2, st 3 & $x$-direction & $-0.1 \leq 0 \leq+0.1$ \\
2 & $D x 2$ & $t u 6 / t u 5$ & $x$-direction & $-0.1 \leq 0 \leq+0.1$ \\
3 & $D y 1$ & $t u 5 /$ su2, su4 & $y$-direction & $-0.05 \leq 0 \leq+0.05$ \\
4 & $D y 2$ & $t u 4 /$ su2, su4 & $y$-direction & $-0.05 \leq 0 \leq+0.05$ \\
5 & $D y 3$ & $t u 3 /$ su2, su4 & $y$-direction & $-0.05 \leq 0 \leq+0.05$ \\
6 & $D y 4$ & $t u 2 / s u 2$, su4 & $y$-direction & $-0.05 \leq 0 \leq+0.05$ \\
7 & $D x D z 1$ & $t u 6 /$ st 1, st 2 & $x, z$-direction & $-0.03 \leq 0 \leq+0.03$ \\
8 & $D z 1$ & $t u 6 / t u 5$ & $z$-direction & $-0.01 \leq 0 \leq+0.01$ \\
\hline
\end{tabular}

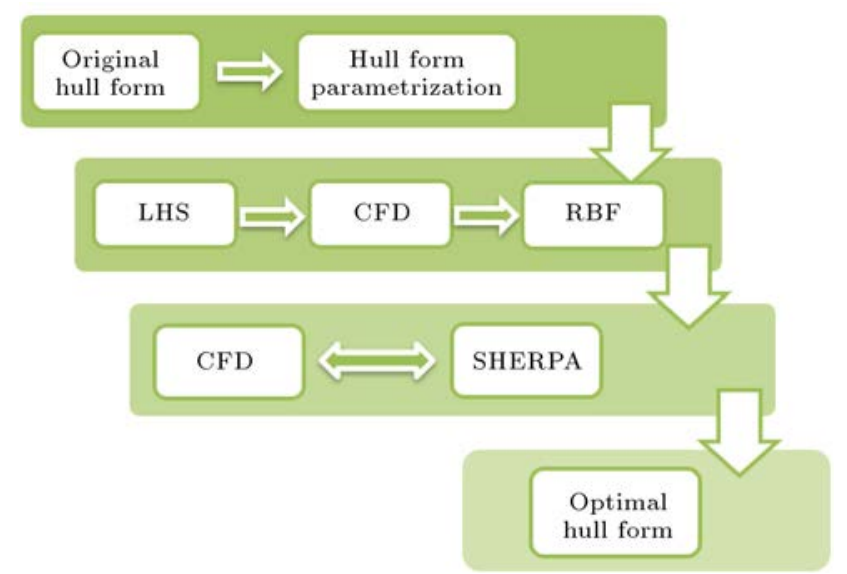

Figure 2. Flowchart of simulation-based optimization framework.

final selected design was introduced into the SHERPA optimizer as an initial run. This platform allows the saving of previous run history for new initialization. Figure 2 illustrates the flow chart of the simulationbased optimization framework.

\subsection{Hull form parametrization}

Parametrization of ship hull is one of the three optimization process steps. The different techniques and their details are explained in reference [1]. One of the key methods is the ASD technique. The ASD technique defines a control volume outside of the geometry through the B-spline technique. The control volume connects to control points in a spatial directions. When the control points moved, the shape of the relevant areas deformed. The hull parametrization and geometric reconstruction were performed using the Sculptor software. Through the defined connection, the domain mesh file was imported into the Sculptor software and exported to the CFD solver automatically. This capability enables us to save time and to avoid spending time on mesh generation. Another advantage of this capability arises from the fact that the user interruption in each optimization step would not occur. There are seven design variables deployed in the present paper. The definition and range of these design variables are defined in Table 2. Figure 3 shows the

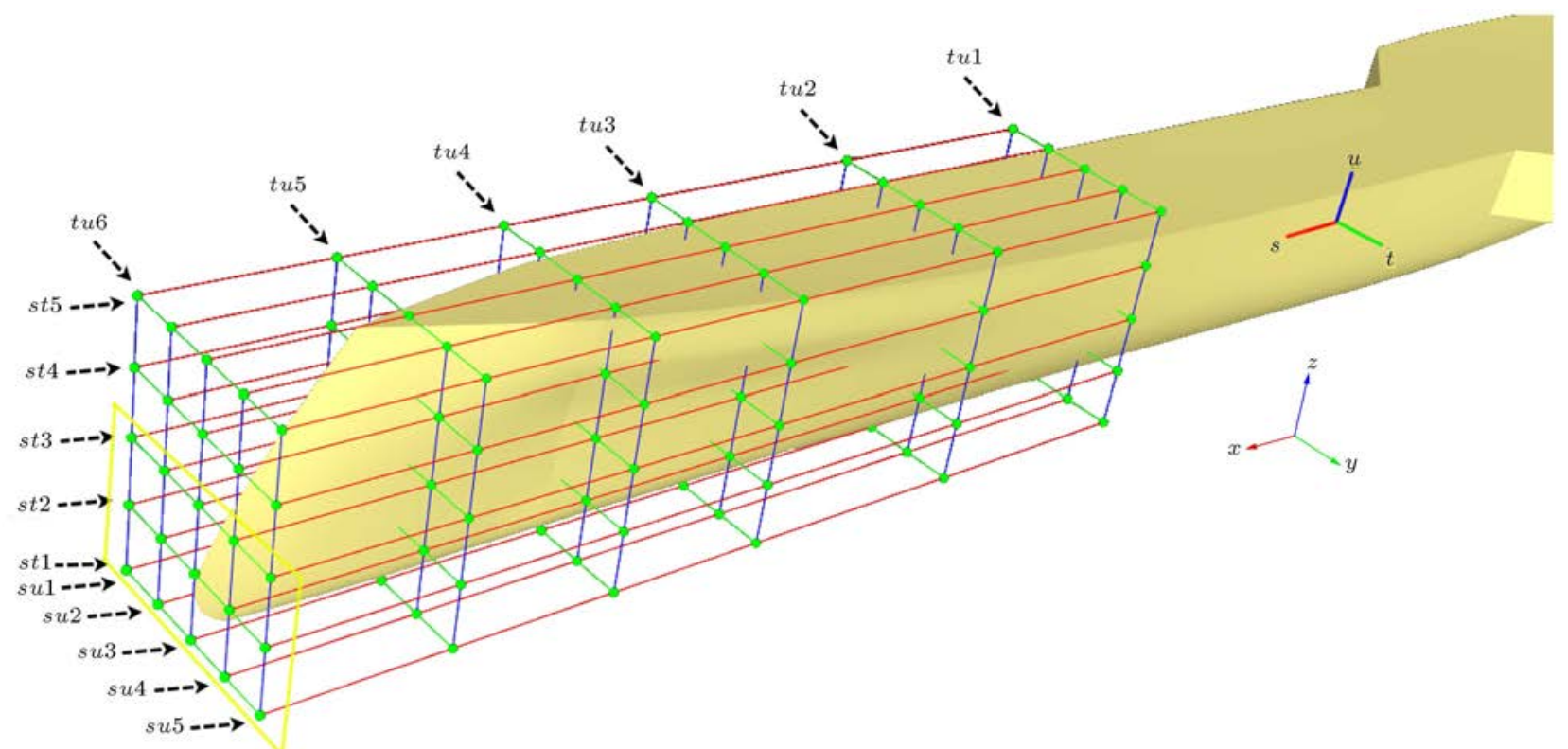

Figure 3. Arbitrary Shape Deformation (ASD) encapsulation around the bow region of the trimaran. 


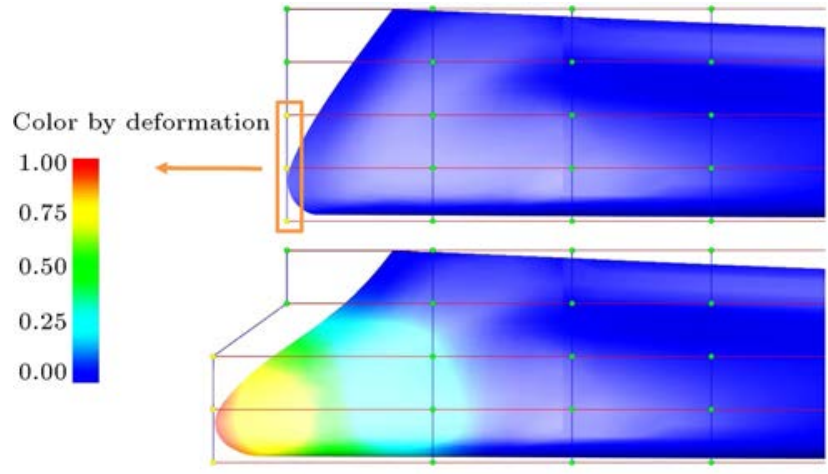

(a)
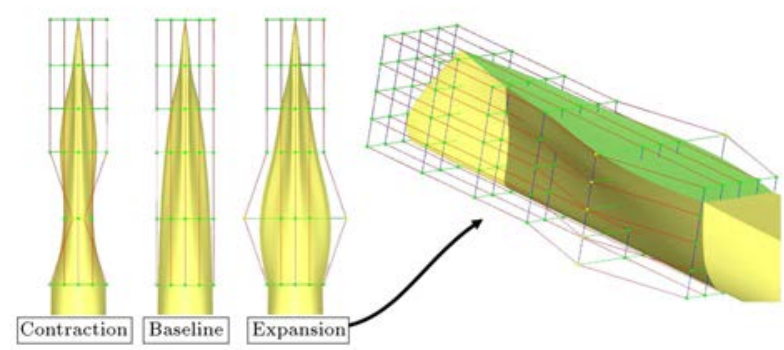

(b)

Figure 4. Bow region deformation for parameter: (a) $D x 1$ for maximum of range $(0.1 \mathrm{~m})$ and $(\mathrm{b})$ parameter Dy4 expansion and contraction.

control point distribution of the $6 \times 5 \times 5$ box. Six sections were distributed along the $x$-direction, and in every section, 5 control points were allocated along $y$ and $z$-direction.

Figure 3 illustrates the ASD encapsulation of the selected control points defined in Table 2 around the bow region of the trimaran. For example, the yellow square in Figure 3 represents the parameter $D x 1$ moving along the $x$-direction. For a movement of $0.1 \mathrm{~m}$, the geometric modification of the above example is shown in Figure 4(a). Also, the expansion and contraction of the section corresponding to the variable Dy 4 are shown in Figure 4(b).

\subsection{Numerical setup}

In this paper, the Reynolds-Averaged Navier-Stokes (RANS) equation solver was used to simulate a trimaran hullform at constant velocity uniform flow. The ship resistance was evaluated by the total drag calculation and its components. A resistance analyses have been carried out for a cruise speed of 16 knot $(1.3 \mathrm{~m} / \mathrm{s}$ for model $)$. The physical model was selected based on the StarCCM+ user guide [40]. The implicit unsteady scheme with a physical time step of $0.02 \mathrm{~s}$ was used for temporal discretization and the SIMPLE algorithm was used to couple the velocity and pressure equations. The RNG $k-\varepsilon$ model was selected as the turbulence model, which has been widely used in industrial applications and similar studies $[13,38]$. To calculate the ship motions, simulation was carried out through the Dynamic Fluid Body Interaction (DFBI) module. The ship is free to move with 2 degrees of freedom of heave and pitch to account for the sinkage and trim.

\subsubsection{Numerical simulation domain}

The box-shaped domain around the hull represents a virtual fluid tank. According to International Towing Tank Conference (ITTC) recommendations, the position of the ship hull in the virtual fluid domain was selected $[41,42]$. To avoid wave reflections, the boundaries of the computational domain were smoothly extended. No-slip wall condition is defined to ship hull surfaces. A prism mesh for the boundary layer of the hull, which had 8 layers and a growth rate of 1.2. was generated. The inlet velocity boundary condition was specified for the inlet, top, and bottom boundaries. The pressure outlet boundary condition was applied to the outlet boundary, and the symmetry plane boundary condition was defined at the domain symmetric plane and side boundary. Figure 5(a) shows the numerical computational domain and the defined boundaries boundary of the trimaran resistance calculation. Furthermore, the boundary distance and the position of the ship model in the virtual towing tank are depicted in Figure 5(b).

\subsubsection{Mesh refinement}

The unstructured trimmer mesh was adopted for mesh operation. To capture the elevation of the free-form surface and the sharp corners of the hull, surface and volumetric refinement were applied to the volume mesh. As shown in Figure 5(c), the volume anisotropic refinement along the $z$-direction was applied near the free surface to maintain a good mesh quality. Therefore, as shown in Figure 5(c), it can be assumed that the mesh refinement pattern around the body can better capture Kelvin waves. To select the appropriate basic size of mesh cell, a mesh was investigated. Mesh refinement and grid convergence continued until the realization of the dependence of the solutions from mesh size. To determine a finer mesh size with acceptable numerical accuracy and an appropriate number of elements, the mesh convergence studies were conducted based on the design speed. The mesh convergence study was conducted out by changing the total resistance value, as shown in Figure 6. The first mesh plan of the mesh study was selected based on the cell size $4 \% \operatorname{Lwl}(\sim 0.125 \mathrm{~m})$. Four mesh plans were developed according to the refinement ratio of $\sqrt{2}$. As shown in Figure 6 and Table 3, the difference between grid 3 and grid 4 is not significant, anymore. Therefore, the full mesh independence has been achieved in grid 3. Taking into account the calculation accuracy and its cost, mesh 3 was selected as the optimum mesh plan. As expressed by the following relation, the relative 


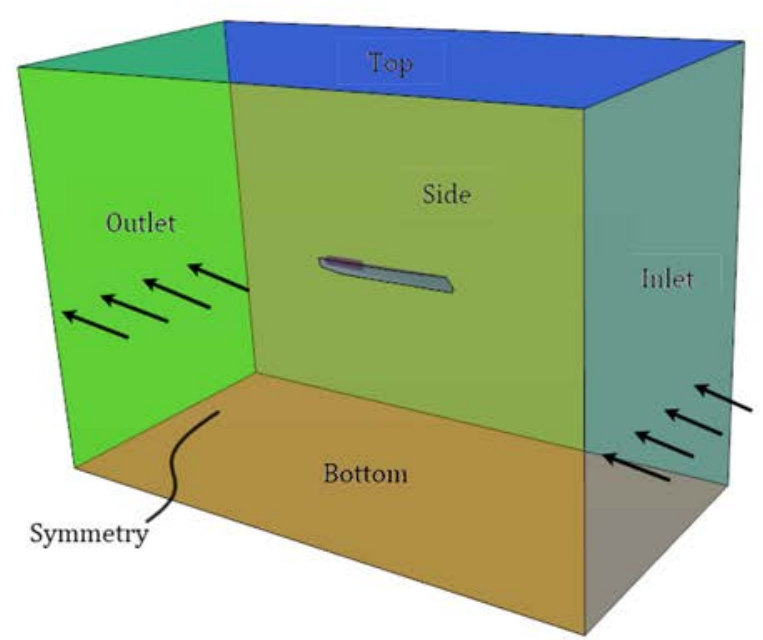

(a)

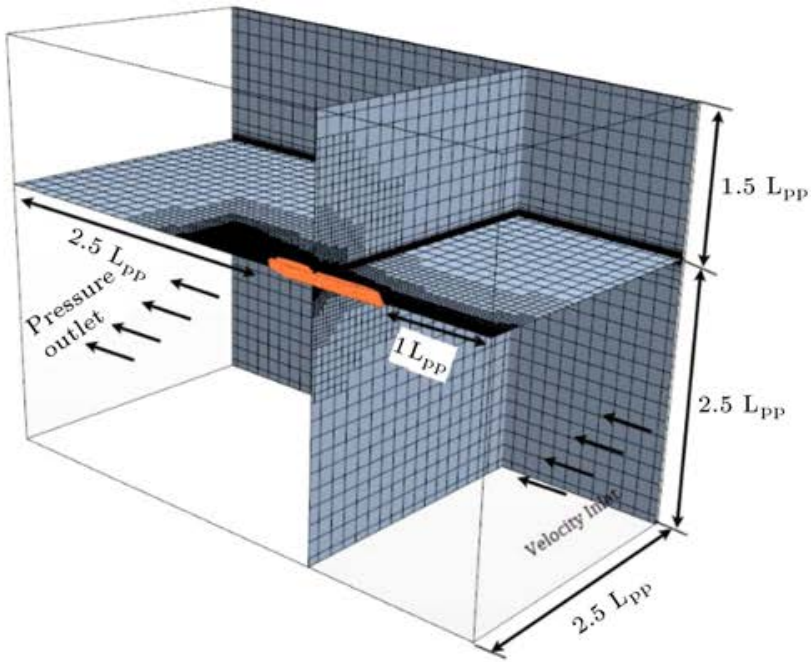

(b)

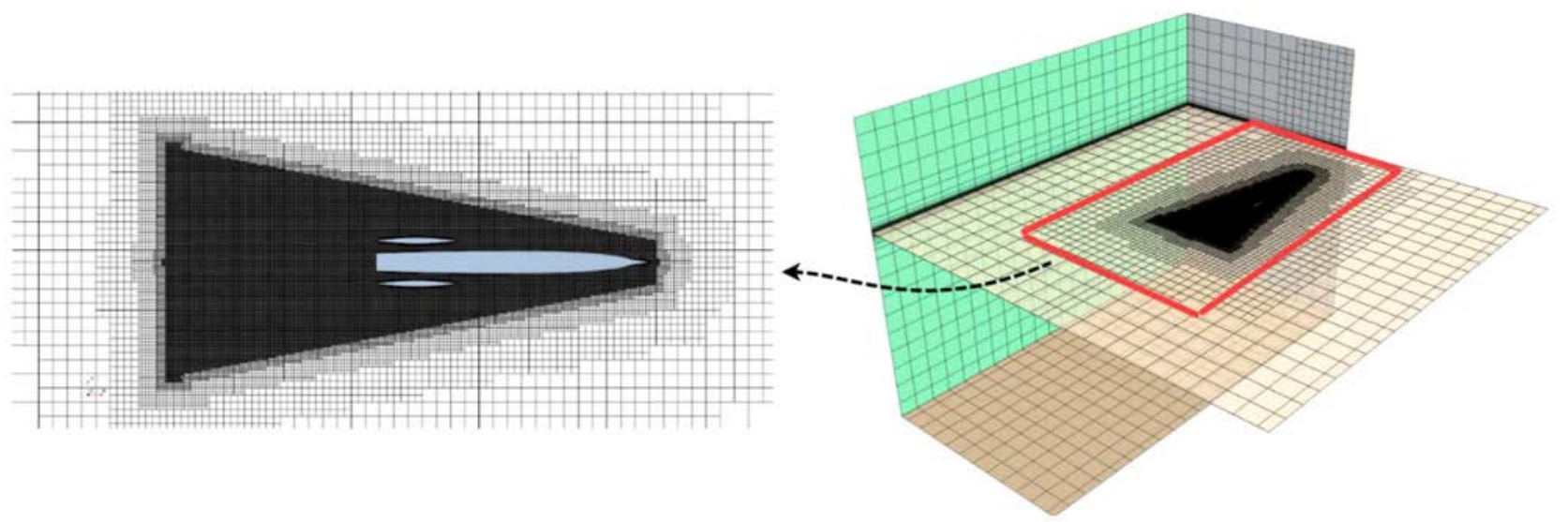

(c)

Figure 5. Numerical simulation characteristics: (a) Computational domain and domain boundaries, (b) boundary distance and ship model location, and (c) unstructured trimmer mesh.

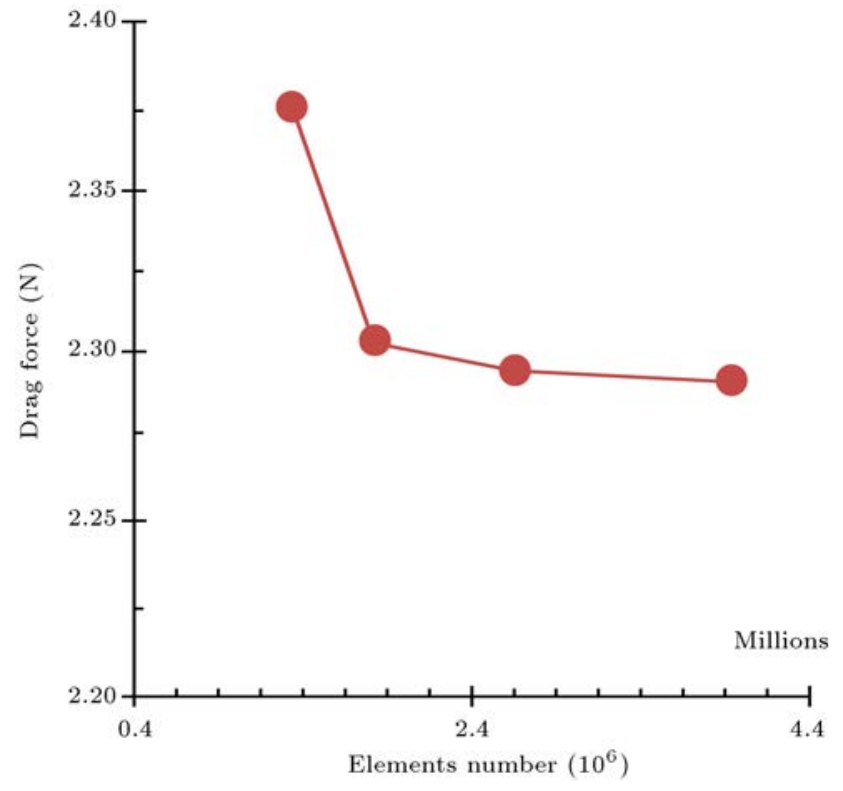

Figure 6. Mesh element number selection. error was calculated to find out the difference between mesh plan results:

$$
\text { Relative error }=\frac{\operatorname{Drag}_{\text {fine }}-\text { Drag }_{\text {finer }}}{\text { Drag }_{\text {fine }}} .
$$

\subsubsection{Uncertainty analysis for mesh study}

The mesh convergence study was conducted by changing the value of total resistance coefficient. According to the refinement ratio of $\sqrt{2}$ presented in the previous section, three mesh plans were made. Mesh plan 1, mesh plan 2, and mesh plan 3 are grid plan formats used for the present uncertainty analysis. Uncertainty analyses have been performed by using the Richardson method $[8,43]$ recommended by ITTC $[44,45]$. According to ITTC recommendations $[44,45]$, uncertainty analysis of iteration $U_{I}$, grid $U_{G}$, time step $U_{T}$, and other parameters $U_{P}$, have been performed for total numerical value of $U_{S N}$.

$$
U_{S N}^{2}=U_{I}^{2}+U_{G}^{2}+U_{T}^{2}+U_{P}^{2} .
$$


Table 3. Mesh independence in different four grid plans.

\begin{tabular}{lcccc}
\hline Item & Grid 1 & Grid 2 & Grid 3 & Grid 4 \\
\hline Basic mesh size (m) & 0.125 & 0.085 & $\mathbf{0 . 0 6}$ & 0.042 \\
Elements number & $1.34 \mathrm{M}$ & $1.82 \mathrm{M}$ & $\mathbf{2 . 6 5} \mathbf{M}$ & $3.94 \mathrm{M}$ \\
Drag force (N) & 2.3625 & 2.2653 & $\mathbf{2 . 2 2 1 2}$ & 2.2156 \\
Relative error & $4.11 \%$ & $1.95 \%$ & $\mathbf{0 . 2 5 \%}$ & - \\
\hline
\end{tabular}

Table 4. Details of simulation condition and drag coefficients of trimaran model.

\begin{tabular}{ccccc}
\hline Model velocity $(\mathbf{m} / \mathbf{s})$ & Grid plan & Total mesh & Time step $(\mathbf{s e c})$ & $\boldsymbol{C}_{\boldsymbol{T}} \times \mathbf{1 0}^{-\mathbf{3}}(\boldsymbol{S})$ \\
\hline \multirow{3}{*}{$1.3 \mathrm{~m} / \mathrm{s}$} & Grid 3 & $1.235 \mathrm{M}$ & $0.032 \mathrm{sec}$ & 5.36 \\
& Grid 2 & $1.845 \mathrm{M}$ & $0.02 \mathrm{sec}$ & 5.56 \\
& Grid 1 & $2.463 \mathrm{M}$ & $0.01 \mathrm{sec}$ & 5.60 \\
\hline
\end{tabular}

Table 5. Uncertainty analysis of the trimaran model.

\begin{tabular}{cccccccccc}
\hline$\varepsilon_{\mathbf{2 1}} \times \mathbf{1 0}^{-\mathbf{5}}$ & $\varepsilon_{\mathbf{3 2}} \times \mathbf{1 0}^{-\mathbf{5}}$ & $\boldsymbol{R}_{\boldsymbol{G}}$ & $\boldsymbol{\delta}_{\boldsymbol{R} \boldsymbol{E}}^{*} \times \mathbf{1 0}^{-\mathbf{5}}$ & $\boldsymbol{P}_{\boldsymbol{G}}$ & $\boldsymbol{C}_{\boldsymbol{G}}$ & $\boldsymbol{F}_{\boldsymbol{S}}$ & $\boldsymbol{U}_{G \boldsymbol{T}} \times \mathbf{1 0}^{-\mathbf{5}}$ & $\boldsymbol{U}_{\boldsymbol{I}} \times \mathbf{1 0}^{-\mathbf{5}}$ & $\boldsymbol{U}_{S N} \times \mathbf{1 0}^{-\mathbf{5}}$ \\
\hline-4.74 & -25.8 & 0.1834 & 1.065 & 4.89 & 4.452 & 7.9 & 8.41 & 3.2 & 8.93 \\
\hline
\end{tabular}

For the present effort, other parameters have not been considered and uncertainties of grid convergence and time step have been combined. This is due to the unsteady scheme of numerical solutions. Therefore, uncertainty analysis of discretization $\left(U_{G T}\right)$ was introduced. Moreover, the following formula was obtained:

$$
U_{S N}^{2}=U_{I}^{2}+U_{G T}^{2} .
$$

The iterative convergence was assessed by using total resistance coefficient history during the last two periods of oscillation that is about $0.88 \% S_{G 1}\left(S_{G 1}\right.$ is the drag coefficient's value in grid plan 1).

$$
U_{I}=\frac{1}{2\left(\tilde{S}_{\max }-\tilde{S}_{\min }\right)}=3.5 e-5
$$

Tables 4 and 5 respectively show the mesh planning and simulation uncertainty analysis of the trimaran model. The ratio of change between simulations of different grid plan was defined as convergence ratio $\left(R_{G}\right)$ :

$$
R_{G}=\varepsilon_{21} / \varepsilon_{32}=\left(S_{2}-S_{1}\right) /\left(S_{3}-S_{2}\right) .
$$

The values of $S$ are the outputs of the numerical simulation in a defined grid plan, which is the resistance coefficient. The $R_{G}$ (grid convergence factor) is less than 1, which means that the convergence of the grid is monotonic. Therefore, grid uncertainty can be estimated through generalized RE (Richardson Extrapolation) $[44,45]$ :

$$
\delta_{R E}^{*}=\frac{\varepsilon_{21}}{r^{P_{G}-1}},
$$

where $P_{G}$ and $r$ is the order of accuracy and refinement ratio $(\sqrt{2})$, respectively. The expression of $P_{G}$ is:
Table 6. Grid verification.

\begin{tabular}{ccc}
\hline$U_{G T}\left(\% \boldsymbol{S}_{\mathbf{1}}\right)$ & $\boldsymbol{U}_{\boldsymbol{I}}\left(\boldsymbol{\%} \boldsymbol{S}_{\mathbf{1}}\right)$ & $\boldsymbol{U}_{S N}\left(\% \boldsymbol{S}_{\mathbf{1}}\right)$ \\
\hline $1.49 \%$ & $0.57 \%$ & $1.58 \%$ \\
\hline
\end{tabular}

$$
P_{G}=\frac{\ln \left(\varepsilon_{32} / \varepsilon_{21}\right)}{\ln (r)} .
$$

Also, the correction factor can be expressed as:

$$
C_{G}=\frac{r^{P_{G}}-1}{r^{P_{G_{e s t}}-1}}
$$

Grid verification based on drag coefficient is summarized in Table 6 . Since the order of accuracy $P_{G}$ is 4.89 , which is sufficiently larger than theoretical value $\left(P_{G_{\text {est }}}=2\right)$ and $C_{G}=4.45$ is sufficiently greater than 1 , the solutions are not in the asymptotic range. The value of $F_{S}$ was calculated by the following equation:

$$
F_{S}= \begin{cases}9.6\left(1-C_{G}\right)^{2}+1.1 & \left|1-C_{G}\right|<0.125 \\ 2\left|1-C_{G}\right|+1 & \left|1-C_{G}\right| \geq 0.125\end{cases}
$$

Uncertainty of the grid and time step $U_{G T}$ was calculated as follows:

$$
U_{G T}=F_{S}\left|\delta_{R E}^{*}\right| .
$$

According to Eq. (5), since the numerical simulation uncertainty $U_{S N}$ was small $\left(1.58 \% S_{G 1}\right)$, it is feasible to capture the differences of $C_{T}$ between the different ship hulls generated by the optimization algorithms in the rest of the paper.

\subsubsection{Validation and verification DTMB5415}

Verification of CFD calculation of ship resistance is performed in this section for 5.72-meter long DTMB5415 
Table 7. Details of simulation condition and drag coefficients of DTMB5415 model.

\begin{tabular}{ccccccc}
\hline $\begin{array}{c}\text { Model velocity } \\
(\mathbf{m} / \mathbf{s})\end{array}$ & Grid plan & Total mesh & $\begin{array}{c}\text { Time step } \\
(\mathbf{s e c})\end{array}$ & $\begin{array}{c}\boldsymbol{C}_{\boldsymbol{T}} \times \mathbf{1 0}^{-\mathbf{3}} \\
(\mathbf{C F D})\end{array}$ & $\begin{array}{c}\boldsymbol{C}_{\boldsymbol{T}} \times \mathbf{1 0}^{-\mathbf{3}} \\
(\mathbf{E F D})\end{array}$ & {$\left[\boldsymbol{C}_{\boldsymbol{T}}\right] \boldsymbol{\%}_{\boldsymbol{D}}$} \\
\hline \multirow{3}{*}{$3.071 \mathrm{~m} / \mathrm{s}$} & Grid 3 & $1.145 \mathrm{M}$ & $0.032 \mathrm{sec}$ & 6.315 & & 5.32 \\
& Grid 2 & $1.633 \mathrm{M}$ & $0.02 \mathrm{sec}$ & 6.563 & 6.67 & 1.60 \\
& Grid 1 & $2.318 \mathrm{M}$ & $0.01 \mathrm{sec}$ & 6.607 & & 0.94 \\
\hline
\end{tabular}

Table 8. Uncertainty analysis of the DTMB 5415 model.

\begin{tabular}{cccccccccc}
\hline$\varepsilon_{\mathbf{2 1}} \times \mathbf{1 0}^{-\mathbf{5}}$ & $\varepsilon_{\mathbf{3 2}} \times \mathbf{1 0}^{-\mathbf{5}}$ & $\boldsymbol{R}_{\boldsymbol{G}}$ & $\boldsymbol{\delta}_{\boldsymbol{R} \boldsymbol{E}}^{*} \times \mathbf{1 0}^{-\mathbf{5}}$ & $\boldsymbol{P}_{\boldsymbol{G}}$ & $\boldsymbol{C}_{\boldsymbol{G}}$ & $\boldsymbol{F}_{\boldsymbol{S}}$ & $\boldsymbol{U}_{G \boldsymbol{T}} \times \mathbf{1 0}^{-\mathbf{5}}$ & $\boldsymbol{U}_{\boldsymbol{I}} \times \mathbf{1 0}^{-\mathbf{5}}$ & $\boldsymbol{U}_{S N} \times \mathbf{1 0}^{-\mathbf{5}}$ \\
\hline-4.4 & -24.8 & 0.1774 & 0.95 & 4.99 & 4.637 & 8.27 & 7.85 & 3.2 & 8.6 \\
\hline
\end{tabular}

model $[46,47]$. The computational domain and boundary distance characteristics were considered the same as in the previous section. The simulation was performed at a model speed of $3.071 \mathrm{~m} / \mathrm{s}$ which is equal to $F_{n}=$ 0.41. Table 7 summarizes the detailed information of the simulated conditions and their difference with the experimental data. The difference between CFD and Experimental Fluid Dynamics (EFD) results was calculated as follows:

$$
D \% \text { Difference }=(E F D-C F D) / E F D \times 100 .
$$

Grid plan 1 is the fine mesh of the study with a difference of $0.94 \%$ in $C_{T}$. These findings and the results of the comparison represent the accuracy and reliability of the numerical analysis strategy. The generated waves around the DTMB5415 hull is shown for $F n=0.41$ in Figure 7 . To accomplish a proper simulation, uncertainty analysis was performed for the present work similar to the previous section. The iterative convergence was evaluated by using total resistance coefficient history during the last two periods of oscillation that is about $0.24 \% D$ ( $D$ is the drag's coefficient value of experiment data). Uncertainty analysis of simulations for the DTMB5415 model is depicted in Table 8 . In addition, grid verification based on the drag coefficient is presented in Table 9 .
Table 9. Grid verification of DTMB 5415.

\begin{tabular}{ccc}
\hline$U_{G T}(\% \boldsymbol{D})$ & $\boldsymbol{U}_{\boldsymbol{I}}(\boldsymbol{\%} \boldsymbol{D})$ & $\boldsymbol{U}_{S N}(\boldsymbol{\%} \boldsymbol{D})$ \\
\hline $2.11 \%$ & $0.24 \%$ & $2.31 \%$ \\
\hline
\end{tabular}

The order of accuracy $P_{G}$ is 4.99 , which is sufficiently larger than theoretical value $\left(P_{G_{e s t}}=2\right)$ and $C_{G}=$ 4.637, are sufficiently larger than 1 ; as it is clear from these findings the solutions are not in the asymptotic range. Since the value of the simulation uncertainty is very small $(2.31 \% D)$, the reliability of the mesh convergence could be achieved.

\subsubsection{RANS governing equation}

The Finite Volume Method (FVM) relating to the STAR-CCM+ software was used to solve the RANS equation. The governing equations of continuity and momentum conservation for three-dimensional incompressible flow were expressed as follows:

$$
\begin{aligned}
\frac{\partial u_{i}}{\partial x_{i}}=0 & \\
\frac{\partial u_{i}}{\partial t}+\frac{\partial}{\partial x_{i}}\left(u_{i} u_{j}\right)= & -\frac{1}{\rho} \frac{\partial p}{\partial x_{i}}+\mu \frac{\partial}{\partial x_{j}}\left(\frac{\partial u_{i}}{\partial x_{j}}+\frac{\partial u_{j}}{\partial x_{i}}\right) \\
& +\frac{\partial}{\partial x_{j}}\left(-\overline{u_{i}^{\prime}} u_{j}^{\prime}\right)
\end{aligned}
$$

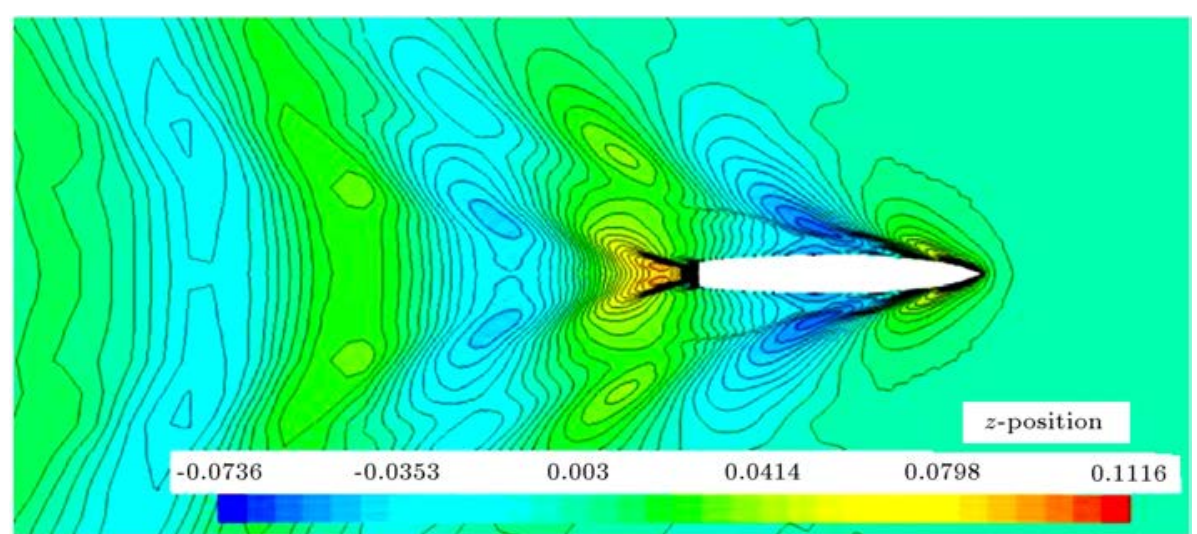

Figure 7. Wave propagation around DTMB5415 hull at $F n=0.41$. 
where $p$ is the mean pressure, $\rho$ is the density and $\mu$ is the kinematic viscosity of the fluid, while velocity $u_{i}$ can be decomposed into mean velocity $\bar{u}_{i}$ and fluctuating velocity $u_{i}^{\prime}$ :

$$
u_{i}=\bar{u}_{i}+u_{i}^{\prime}
$$

For optimization problems that require a large number of CFD operations, the choice of a cost-effective and efficient turbulence model is more critical. Therefore, the $k-\varepsilon$ turbulence model was applied because it has less CPU time than other models. Reynolds stress tensor was calculated as follows:

$$
\overline{u_{i}^{\prime} u_{j}^{\prime}}=-\mu_{t}\left(\frac{\partial u_{i}}{\partial x_{j}}+\frac{\partial u_{j}}{\partial x_{i}}\right)+\frac{2}{3} \delta_{i j} k,
$$

where, $\mu_{t}$ is eddy viscosity and could be calculated as follows:

$$
\mu_{t}=C_{\mu} \frac{k^{2}}{\varepsilon}
$$

$C_{\mu}$ is the empirical constant $\left(C_{\mu}=0.09\right)$ and $k$ is the turbulent kinetic energy and $\varepsilon$ is the turbulent dissipation rate. Transport equations were solved for $k$ (Eq. (19)) and $\varepsilon$ (Eq. (20)):

$$
\begin{aligned}
& \frac{\partial k}{\partial t}+\frac{\partial\left(k u_{j}\right)}{\partial x_{j}}=\frac{\partial}{\partial x_{j}}\left[\left(\mu+\frac{\mu_{t}}{\sigma_{k}}\right) \frac{\partial k}{\partial x_{j}}\right]+P_{k}-\varepsilon \\
& \frac{\partial \varepsilon}{\partial t}+\frac{\partial\left(k u_{j}\right)}{\partial x_{j}}=\frac{\partial}{\partial x_{j}}\left[\left(\mu+\frac{\mu_{t}}{\sigma_{\varepsilon}}\right) \frac{\partial \varepsilon}{\partial x_{j}}\right]+C_{\varepsilon 1} P_{k} \frac{\varepsilon}{k} \\
& -C_{\varepsilon 2} \frac{\varepsilon^{2}}{k}
\end{aligned}
$$

More explanation can be found in [48].

\subsubsection{Computational time and platform}

The optimization process has been implemented in the Computer Center of the Maritime School of Amir Kabir University of Technology (AUT). On a PC (2.8 up to $3.8 \mathrm{GHz}$ Intel囚, 16 GB RAM, 8-core SSD hard drive), the total computing time required to obtain a direct optimization solution and build an alternative model on the PC is 48 days. All simulations were performed automatically, and the user only needs to check the mesh quality every five times.

\subsection{Surrogate model construction}

The implementation of surrogate models can speed up the optimization process. The surrogate model aims to describe the relationship between the optimization goal and the variables used. Basic steps were required to construct a surrogate model for design research:

1. Define sample point by using LHS;

2. Evaluate the sobjective function for generated sample designs by CFD;
3. Construct a surrogate model through the RBF method.

The LHS technique was adopted to distribute 40 design points in the design space for generating data. Subsequently, the total resistance of these hull forms was calculated. All feasible sample designs are shown in Figure 8. Consequently, inputs and outputs were prepared to fit a surrogate model. In comparison to the cost of acquiring the data, the acquired model can be developed without incurring large expenses. Following the construction of the RBF surrogate model, crossvalidation was performed to examine the accuracy of the model. To determine a sample point and to construct a surrogate model by the remaining sample points, cross-validation was carried out. The CFD tool computes the difference between the exact values of the objective function at a given sample point, and the RBF surrogate model predicts the approximate value of the objective function at a given sample point. This RBF surrogate model was together with other subset of the training set [30]. If the difference is small enough, the constructed model is valid. Otherwise, the number of sampling points increased, and the CFD calculation for new samples repeated. Besides, the infeasible design indicated that the displacement of the ship hulls changed by more than $1 \%$. Figure 9 shows the cross-validations of these models. A parallel data plot is depicted in Figure 10 which also gives a high-level graphical view of the relationships between multiple variables and responses. This allows us to determine the minimum value used to compare the design aspects of different design sets. The best design set is shown in Figure 10 and is marked as a continuous black line.

As is shown in Figure 10, the optimum designed resistance is $2.05 \mathrm{~N}$. This design was introduced to the direct optimization process. To study the effectiveness of the variables, the correlation diagram is shown in the Figure 11. Values in the cell were based on Pearson's correlation coefficient, which illustrate the statistical relationship, or association, between two continuous

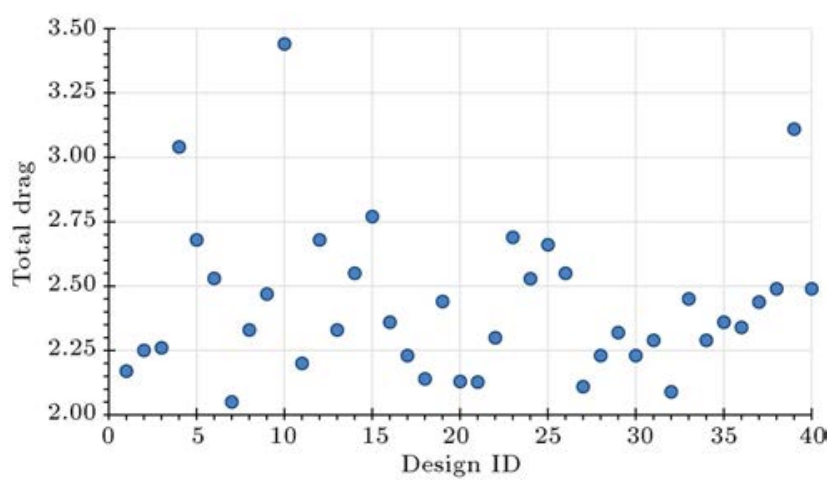

Figure 8. Feasible design samples made by Latin Hypercube Sampling (LHS). 


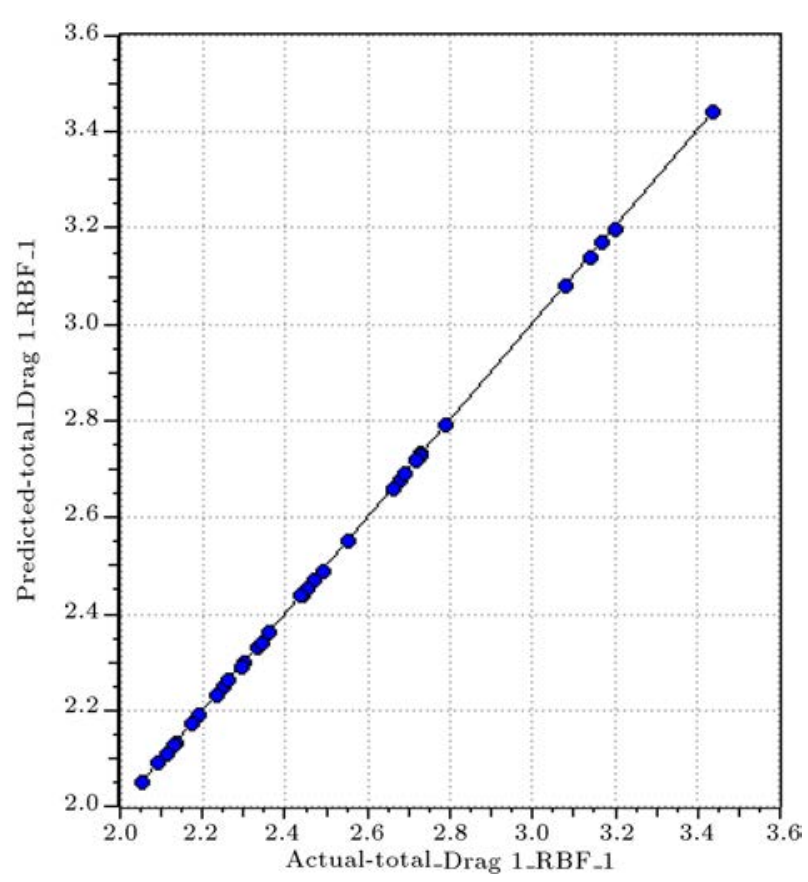

Figure 9. Cross validations of the surrogate model.

variables. The coefficient value ranges from +1 to -1 , where +1 represents a perfect positive relationship, while -1 represents a perfect negative relationship, and no relationship was specified by the value 0 . Coefficient values between \pm 0.50 and \pm 1 indicate a strong correlation, while values between \pm 0.30 and \pm 0.49 show medium correlations and values below \pm 0.29 specify small correlation. The most critical parameter in this study is $D x 1$, which changes the forward region of the bow. Its calculated value is
-0.38 , indicating a moderate total resistance effect. Therefore, the wave-piercing bow should be extended in positive $x$-direction to reduce the drag. The three important parameters for firmly changing the bow shape are $D x 1, D x 2$, and $D x D z 1$. The $3 \mathrm{D}$ response surface results of the RBF surrogate model of these three parameters are shown in Figure 12. It should be noted that the increase in $D x 1$ and the decrease in $D x 2$ cause as strong a drag reduction as possible. It should also be noticed that displacement constraint does not allow arbitrary manipulation of the hull form. The shape of the inverted bow after the maximum extension of $D x 1, D x 2$, and $D x D z 1$ variables in the optimum direction is shown in Figure 13. Hence, one can conclude that the rake angle in the forefoot region, the sharper inverted bow, and the appropriate curve extension; reduce the resistance of this type of bow.

\subsection{Direct optimization SHERPA}

The SHERPA optimization algorithm is a novel searchbased method that selects the best attributes of each search method proposed by StarCCM [40]. This method also proposes a suitable solution in the singleobjective optimization process. The defined input variable is the same defined in Subsection 2.2, and the initial hullform selects from the best output of Subsection 2.4. The direct optimization SHERPA algorithm explores different designs aimed at minimizing the total ship resistance. The history diagram of 40 designs is shown in Figure 14. The optimization process continued until no significant drag reduction was observed. The Initial design drag was about

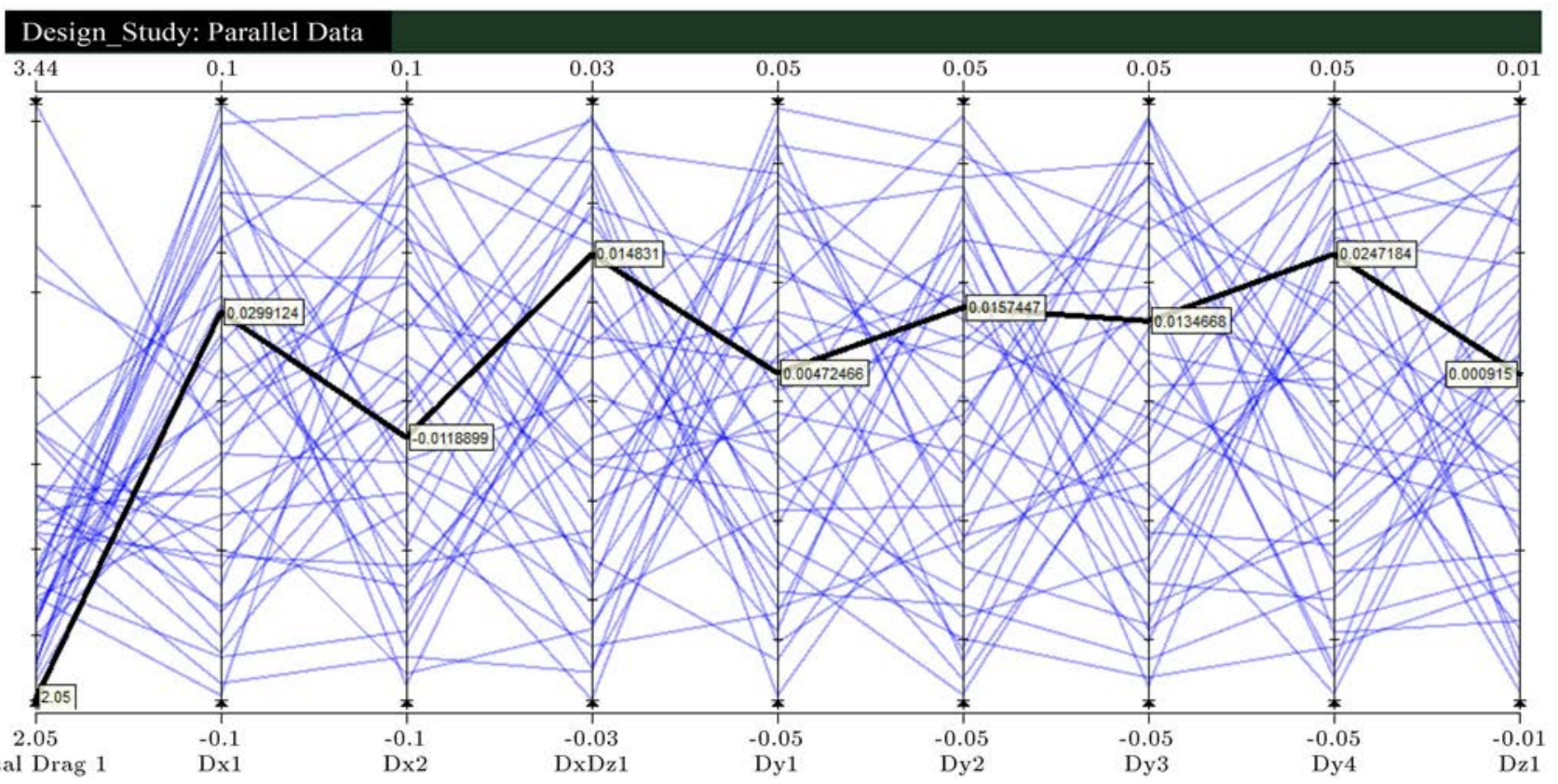

Figure 10. Parallel data plot for various values of inputs. 


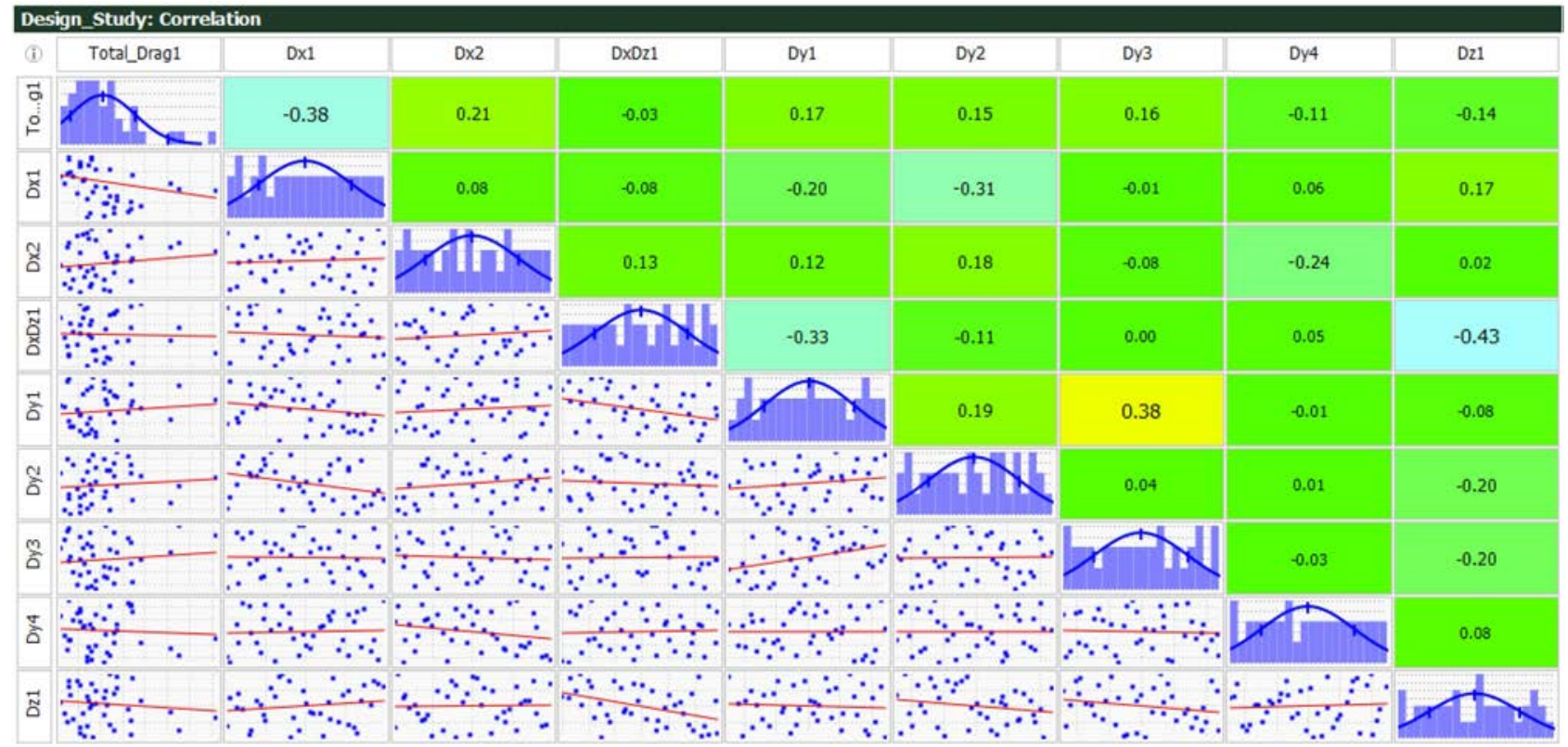

Figure 11. Correlation study between design variables and response.

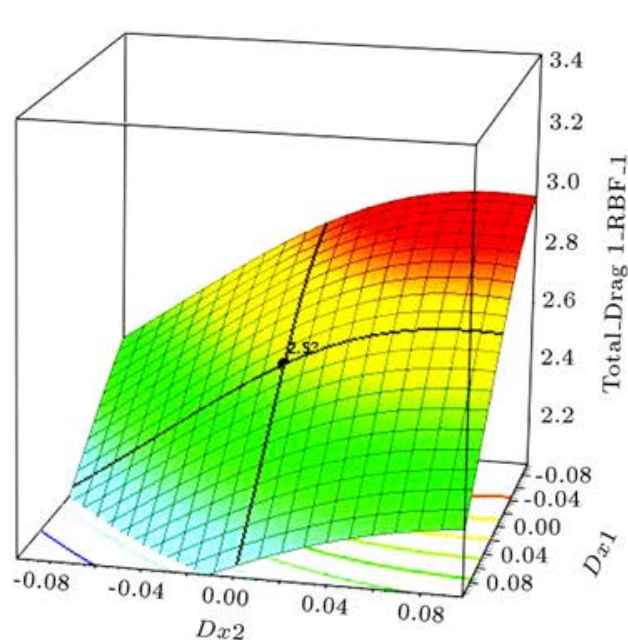

(a)

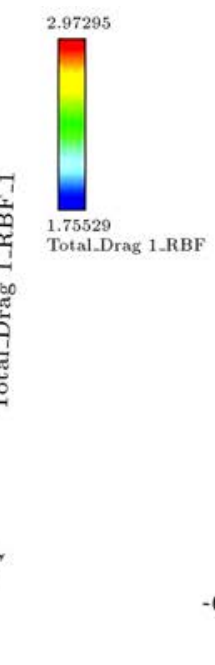

Figure 12. 3D response surface for:
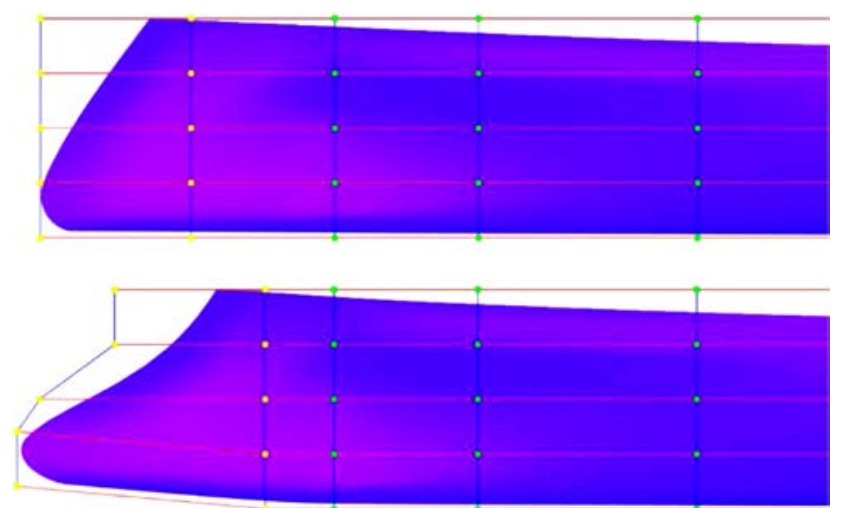

Figure 13. Changing variables $D x 1, D x 2$, and $D x D z 1$ at maximum level.

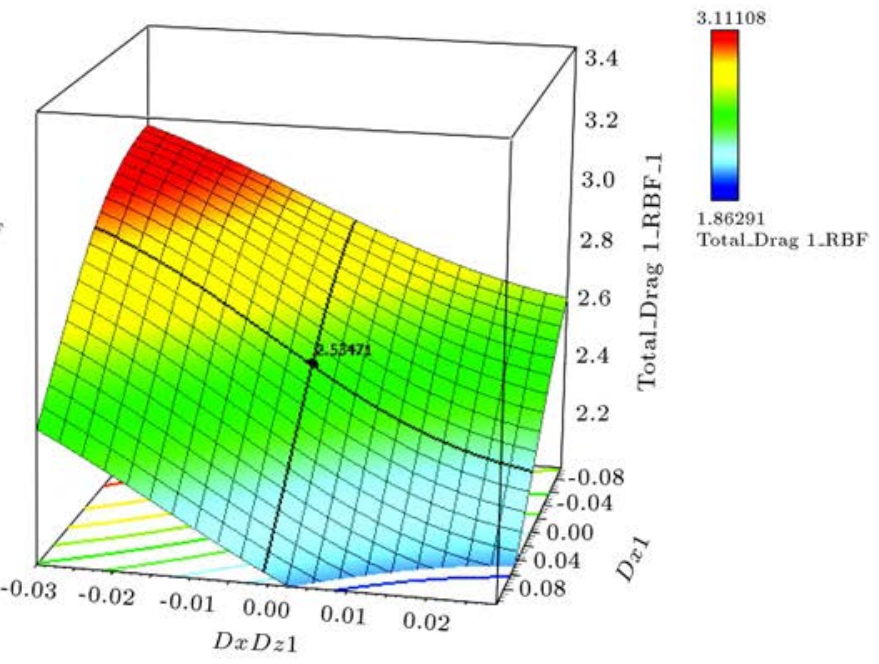

(b)

a) $D x 1-D x 2$ and (b) $D x 1-D x D z 1$.

$2.05 \mathrm{~N}$, while the final optimized ship hull drag was determined to be $1.98 \mathrm{~N}$.

\section{Results and comparison}

After combining two different optimization techniques, the final optimum trimaran hull was finally obtained. In this section, the achieved ship hull form and the original form were compared. Table 10 shows the geometric parameter changes of the hull, and Table 11 shows the calculated drag reduction at the speeds of 16,20 , and 25 knots. It was observed that for the knots with speeds of 16, 20, and 25, the total resistance was reduced by about $10.2 \%, 2.65 \%$, and 


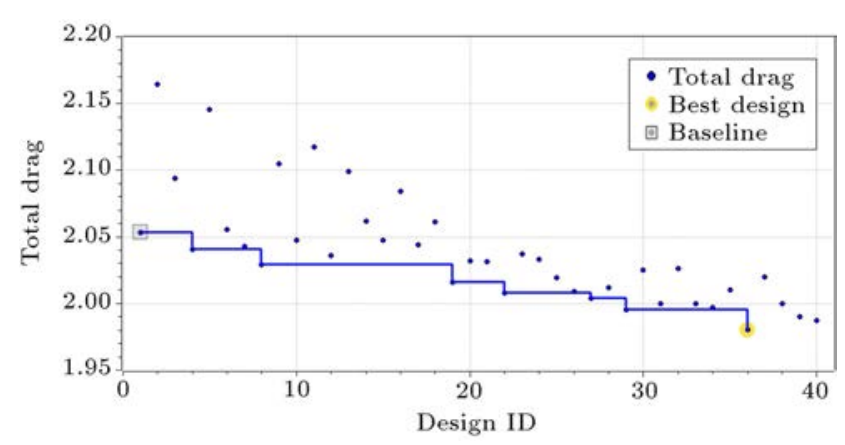

Figure 14. History diagram of the optimization process.

Table 10. Optimum value of the design variables.

\begin{tabular}{ccc}
\hline No. & Parameter & Value \\
\hline 1 & $D x 1$ & 0.0358 \\
2 & $D x 2$ & -0.0116 \\
3 & $D y 1$ & 0.005 \\
4 & $D y 2$ & 0.0102 \\
5 & $D y 3$ & 0.0125 \\
6 & $D y 4$ & 0.0023 \\
7 & $D x D z 1$ & 0.0226 \\
8 & $D z 1$ & 0.0006 \\
\hline
\end{tabular}

$0.293 \%$ respectively (see also Figure 15 ), which also indicates the effect of the new bow on the resistance reduction.

Table 12 compares the components of the total resistance which are the shear and the pressure resistance. The table shows the value of each drag sepa-

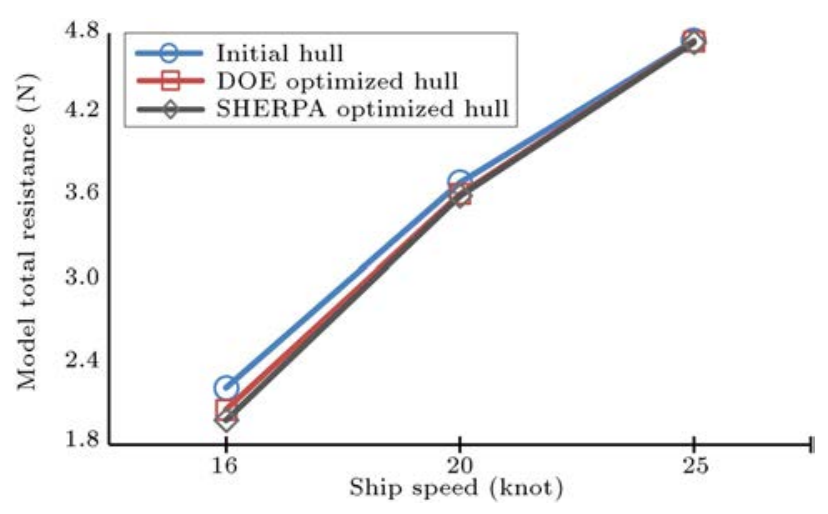

Figure 15. Resistance change for different speeds.

rately. The contribution rate of the pressure resistance of the initial hull is $23.66 \%$. For the optimized hull, the change is $22.8 \%$. Although for an optimized hull, both shear resistance and pressure resistance decreased, the decrease in pressure resistance is higher than the shear resistance.

Figure 16 shows the wave shape of the initial hull and optimized hull computed at a speed of 16 knots. Figure 17 shows similar characteristics to Figure 16, but the difference between these two figures is the range of the $Z$ position legend. As observed, the new bow shape of the optimum hull reduces the bow and shoulder waves and their amplitudes, and these results lead to a reduction in total resistance. Figure 18 shows a comparison of the free surface elevation of the initial hull and the optimal hulls and Figure 19 shows the

Table 11. Trimaran ship hull specifications: Initial and optimized vessel changes.

\begin{tabular}{lccccc}
\hline \multicolumn{1}{c}{ Specifications } & Initial hull & $\begin{array}{c}\text { Optimized } \\
\text { hull DOE }\end{array}$ & Change \% & $\begin{array}{c}\text { Optimized } \\
\text { hull SHERPA }\end{array}$ & Change \% \\
\hline Displacement (kg) & $36.0 \mathrm{~kg}$ & $35.89 \mathrm{~kg}$ & 0.305 & $35.87 \mathrm{~kg}$ & 0.361 \\
Resistance at 16 kn (N) & 2.2159 & 2.0566 & 7.19 & 1.9901 & 10.2 \\
Resistance at 20 kn (N) & 3.7108 & 3.6254 & 2.30 & 3.6125 & 2.65 \\
Resistance at 25 kn (N) & 4.7460 & 4.7382 & 0.165 & 4.7321 & 0.293 \\
\hline
\end{tabular}

Table 12. Resistance component comparison between final optimized and initial hull.

\begin{tabular}{|c|c|c|c|c|c|c|}
\hline \multirow{2}{*}{ Resistance components } & \multicolumn{3}{|c|}{ Initial } & \multicolumn{3}{|c|}{ Optimized } \\
\hline & Total & Shear & Pressure & Total & Shear & Pressure \\
\hline Resistance at $16 \mathrm{kn}(\mathrm{N})$ & 2.2159 & 1.6916 & 0.5242 & 1.9901 & 1.5363 & 0.4537 \\
\hline Resistance at $20 \mathrm{kn}(\mathrm{N})$ & 3.7108 & 2.8053 & 0.9054 & 3.6125 & 2.7382 & 0.8742 \\
\hline \multirow[t]{3}{*}{ Resistance at $25 \mathrm{kn}(\mathrm{N})$} & 4.746 & 3.5652 & 1.1808 & 4.7321 & 3.6025 & 1.1295 \\
\hline & \multicolumn{3}{|c|}{$\begin{array}{c}\text { Quota of total } \\
\text { resistance components } \\
\text { for initial hull }(\%)\end{array}$} & \multicolumn{3}{|c|}{$\begin{array}{c}\text { Quota of total } \\
\text { resistance components } \\
\text { for optimized hull }(\%)\end{array}$} \\
\hline & Total & Shear & Pressure & Total & Shear & Pressure \\
\hline Resistance at $16 \mathrm{kn}(\mathrm{N})$ & - & 76.34 & 23.66 & - & 77.20 & 22.80 \\
\hline Resistance at $20 \mathrm{kn}(\mathrm{N})$ & - & 75.60 & 24.40 & - & 75.80 & 24.20 \\
\hline Resistance at $25 \mathrm{kn}(\mathrm{N})$ & - & 75.12 & 24.88 & - & 76.13 & 23.87 \\
\hline
\end{tabular}




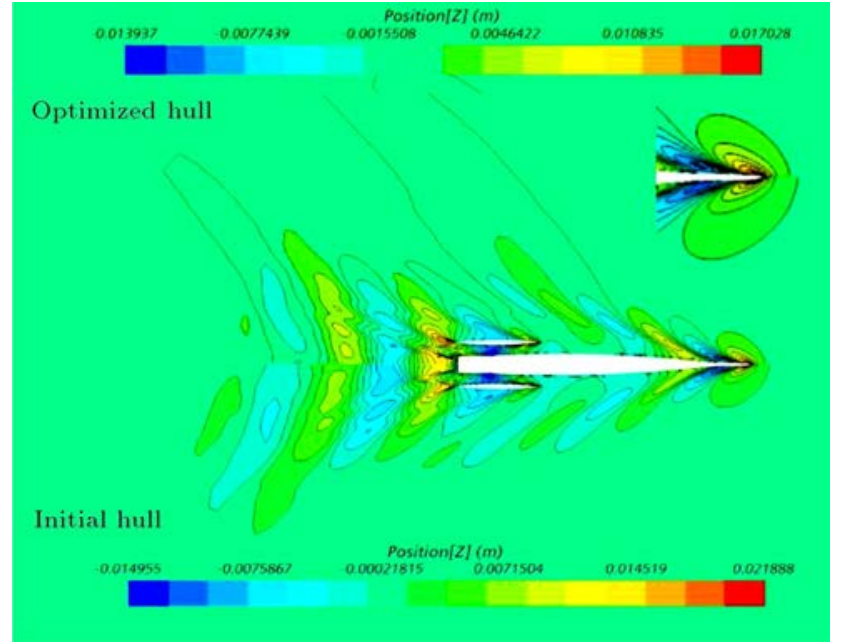

Figure 16. Wave patterns of the initial and the optimized hull at speed 16 knot with free contour range.

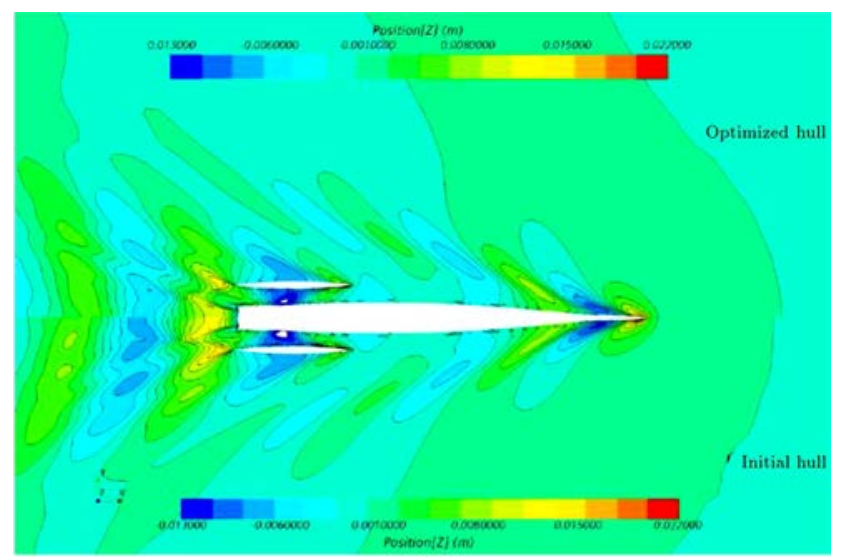

Figure 17. Wave patterns of the initial and the optimized hull at speed 16 knot with fixed contour range.

comparison of the total pressure on the surface of the initial hull and the optimized hull. Figure 19 shows the reduction of the pressure value and the smoothness of its distribution, which led to the reduction of the ship resistance.

The shape of the bow region is significantly changed. The forefoot region moves upward and forms the rake angle of the forefoot. The nose of the bow region became sharper. The obtained optimization results show that the bluntness form for wave-piercing bow has a more suitable performance. As shown in Figure 19, the high- pressure region in the bow region was reduced in improved hullform. To better understand the fluid flow characteristics near the bow region, a comparison of the initial and optimized hullform is shown in Figure 20. A reduction in wave amplitude can be observed in the bow region. Therefore, for the optimized hull, the generation of waves and their propagation to the site was reduced. The amplitude of the bow wave reduces by about $0.006 \mathrm{~m}$ for the model.

\section{Conclusion}

In this paper, based on the connection of the three basic disciplines, an optimization process was developed. The forebody of a wave-piercing bow trimaran has been optimized to reduce resistance in calm waters. A novel and complex trimaran hullform with an inverted bow shape, for which sufficient prior knowledge and information was not available was optimized. Therefore, a Computational Fluid Dynamics (CFD)based optimization problem was defined for the shape modification of the displacement trimaran hull. The first step is to define design variables through the Arbitrary Shape Deformation (ASD) box around the car body, then the role of geometric reconstruction was determined. By using a comprehensive design research tool, the parameterized geometry was introduced to the CFD solver for drag (objective function) evaluation. In this paper, the HEEDS software manager tool was employed to perform the optimization cycle. In the numerical simulation step (second step), the ReynoldsAveraged Navier-Stokes (RANS) equation with $k-\varepsilon$ turbulence model and the VOF method was used. The hydrodynamic optimization was enhanced by combining two different optimization techniques. In the third step, A Latin hypercube sampling distributes the design samples based on an efficient response, and subsequently, an RBF interpolation estimates the

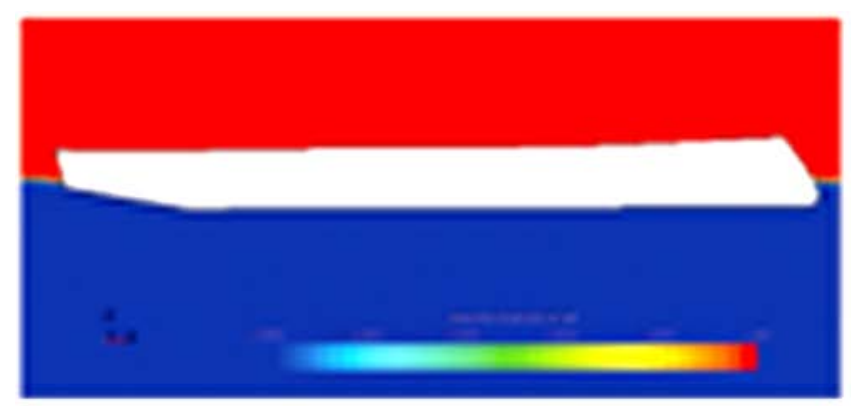

(a)

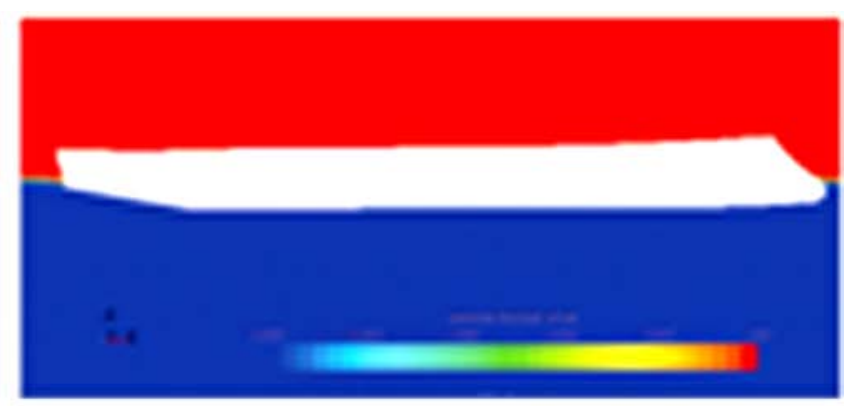

(b)

Figure 18. Free surface elevation of the initial (a) and the optimized (b) hull at speed 16 knot. 


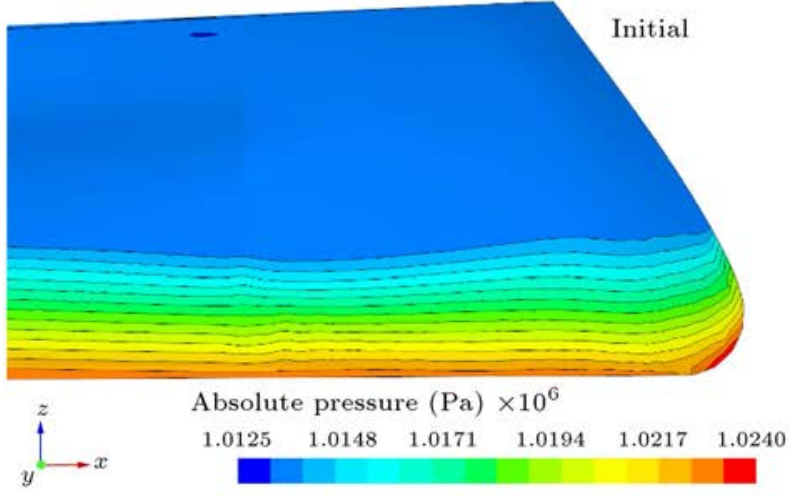

(a)

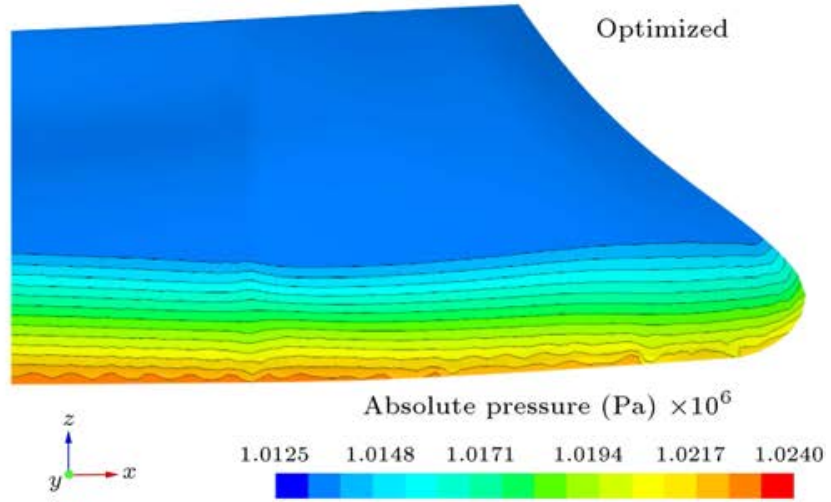

(b)

Figure 19. Absolute total pressure of the initial (a) and the optimized (b) hull at speed 16 knot.

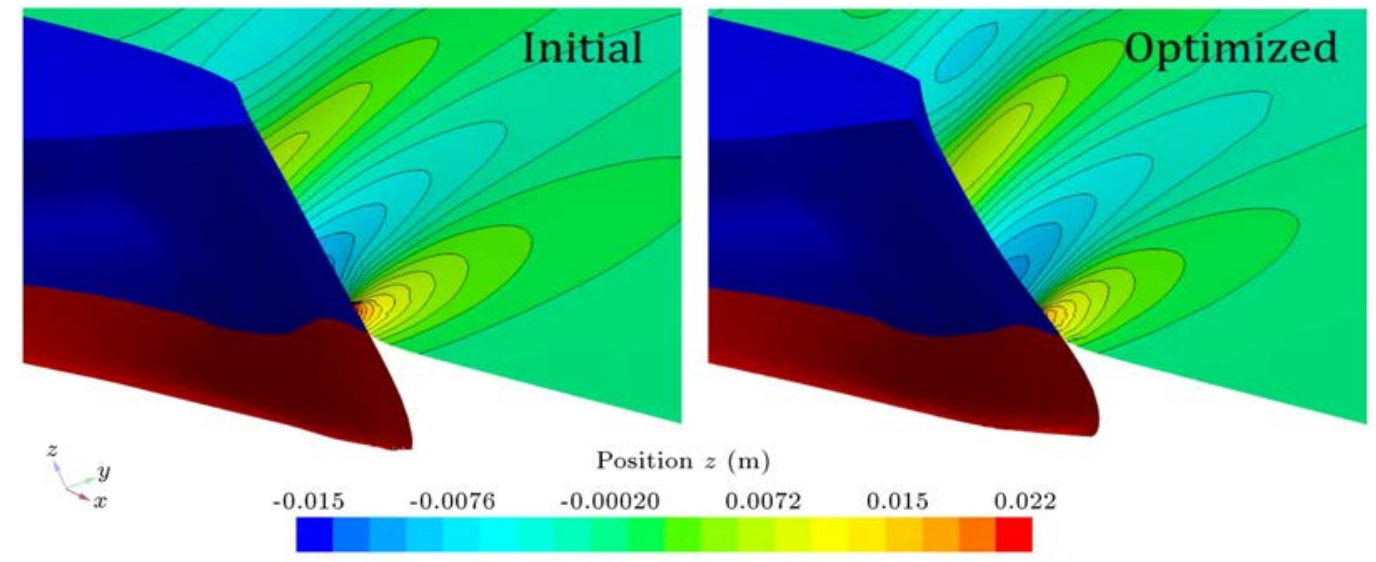

(a)

(b)

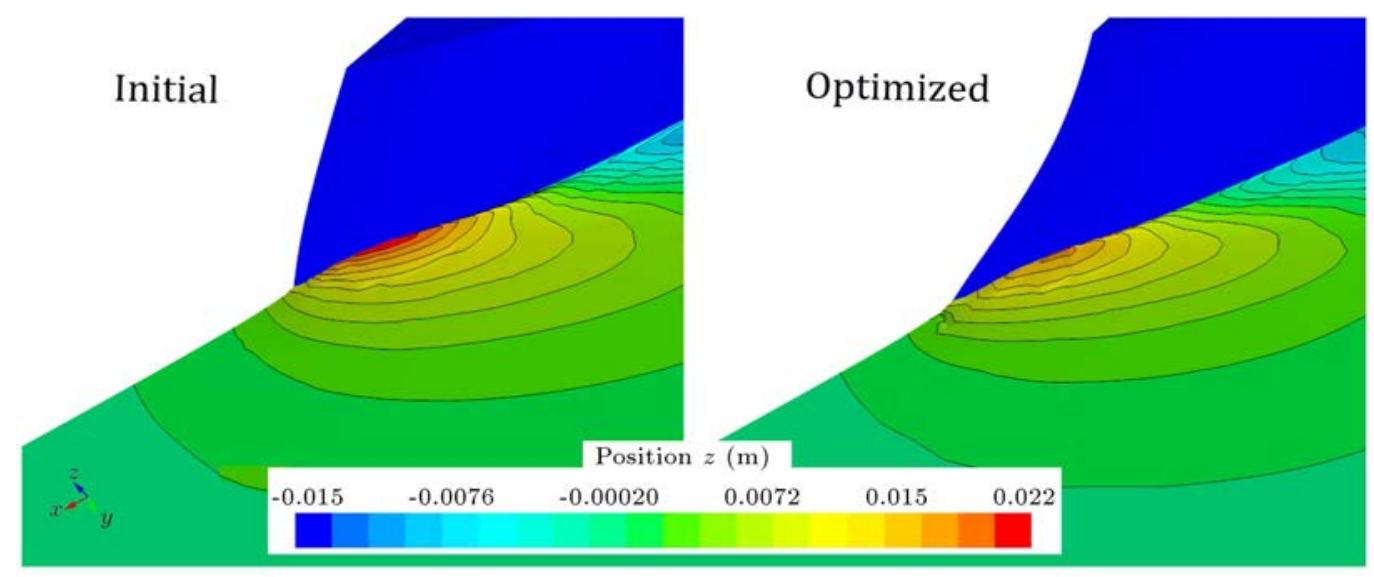

(c)

(d)

Figure 20. Wave pattern on hull and around the hall: (a) Initial hull left view, (b) optimized hull left view, (c) initial hull right view, and (d) optimized hull 10.24200/sci.2020.56644.4833 right view, at speed 16 knot.

response of the system. At the same time, the RBFbased surrogate model approximated the best design and corresponding variables. The final selected design was introduced to SHERPA optimizer as an initial run of direct optimization. Taking into account the small changes in the ship displacement, the appropriate values of the eight design variables was obtained. The total drag reduction at cruising speed was determined to be $10.2 \%$. Consequently, this reduction in resistance significantly reduces fuel, operating costs, and greenhouse gas $\left(\mathrm{NO}_{x}\right.$ and $\mathrm{So}_{x}$ ) emissions. The bow nose became sharper, and the forfeet moved slightly in the 
upward direction. These results showed the changes of the inverted bow. Therefore, the appropriate curve extension from deck to the bluntness of inflated bow led to the reduction of the resistance. Because of deformations in the bow region, reduction of wave amplitude and consequently reduction of local pressure can be observed in the fore region of the hull.

All applied tools and methods have been designed for the reduction of computational time and effort. compared with traditional ship design methods, the method proposed in the present study has considerable advantages and it opens up new ways to use costeffective and high-performance computing resources to achieve higher performance and lower cost designs. The introduction of effective optimization tools is the main purpose of the current paper. The comparison between the initial hull and the optimized hull proves the effectiveness and validity of the proposed optimization design strategy. Based on the obtained results, one can conclude that combining the two CFD-based optimizers and the hull morphing technology is an appropriate method for fluid-exposed geometry optimization with complex geometry in different hydrodynamic problems. The proposed optimization platform can be used in marine industrial applications for hull form optimization. This attempt can be extended to the different speed optimization studies and seakeeping objective function, in future researches.

\section{Acknowledgment}

This research received no specific grant from any funding agency in the public, commercial, or not-forprofit sectors and there is no conflict of interest. Data will be made available, upon request.

\section{Nomenclature}

$\begin{array}{ll}C_{G} & \text { Correction factor } \\ C_{T} & \text { Total resistance coefficient } \\ C_{\mu} & \text { Empirical constant } \\ f(x) & \text { Objective function } \\ F_{S} & \text { Simulation uncertainty factor } \\ F n & \text { Froude number }=\frac{U}{\sqrt{g L_{p p}}} \\ g & \text { Gravitational acceleration } \\ k & \text { Turbulent kinetic energy } \\ L_{p p} & \text { Length between perpendicular } \\ L_{w l} & \text { Length of waterline } \\ N & \text { Number of design variables } \\ P & \text { Pressure } \\ P_{G} & \text { Order of accuracy } \\ P_{\text {Gest }} & \text { Order of accuracy for estimation }\end{array}$

$\begin{array}{ll}r & \text { Refinement ratio } \\ R_{T} & \text { Total resistance } \\ R_{G} & \text { Convergence ratio } \\ S & \text { Subset of feasible space } \\ S_{G} & \text { Drag coefficient's value of grid plans } \\ U & \text { Ship velocity } \\ u_{i} & \text { Fluid velocity } \\ \bar{u}_{i} & \text { Mean velocity } \\ u_{i}^{\prime} & \text { Fluctuating velocity } \\ U_{I} & \text { Uncertainty of iteration } \\ U_{G} & \text { Uncertainty of grid convergence } \\ U_{T} & \text { Uncertainty of time step } \\ U_{P} & \text { Uncertainty of other parameters } \\ U_{G T} & \text { Uncertainty of grid and time step } \\ U_{S N} & \text { Uncertainty of numerical simulation } \\ x & \text { Design variables } \\ \mu_{t} & \text { Eddy viscosity } \\ \varepsilon & \text { Turbulent dissipation rate } \\ \varepsilon_{21} & \text { Difference of the drag coefficient's } \\ \varepsilon_{32} & \text { value between grid plan } 2 \text { and 1 } \\ \nu & \text { Difference of the drag coefficient's } \\ \delta_{R E} & \text { value between grid plan } 3 \text { and } 2 \\ \Delta & \text { Sinematic viscosity } \\ & \text { Ship displacement } \\ & \end{array}$

\section{References}

1. Zhang, B.J. and Zhang, S.L., Research on Ship Design and Optimization Based on Simulation-Based Design (SBD) Technique, Springer Pub. (2018).

2. Wilson, M.B., HSU, C.C., and Jenkins, D.S. "Experiments and predictions of the resistance characteristics of a wave cancellation multihull ship concept", 23rd American Towing Tank Conference, USA, pp. 103-112 (1993).

3. Suzuki, K. and Ikehata, M. "Studies on minimization of wave making resistance for high speed trimaran with small outriggers", J. Soc. Nav. Archit. Japan, 177, pp. 113-122 (1995)

4. Brizzolara, S., Bruzzone, D., and Tincani, E. "Automatic optimization of a trimaran hull form configuration", 8th International Conference on Fast Sea Transportation (FAST05), Saint Petersburg, Russia, pp. 136-145 (2005).

5. Ghadimi, P., Nazemian, A., and Ghadimi, A. "Numerical scrutiny of the influence of side hulls arrangement on the motion of a Trimaran vessel in regular waves through CFD analysis", J. Brazilian Soc. Mech. Sci. Eng., 41(1), p. 1 (2019a). 
6. Jia, J.B., Zong, Z., and Shi, H.Q. "Model experiments of a trimaran with transom stern", Int. Shipbuild. Prog., 56, pp. 119-133 (2009).

7. Wang, S., Ma, S., and Duan, W. "Seakeeping optimization of trimaran outrigger layout based on NSGA-II", Applied Ocean Research, 78, pp. 110-122 (2018).

8. Zong, Z., Hong, Z., Wang, Y., et al. "Hull form optimization of trimaran using self-blending method", Applied Ocean Research, 80, pp. 240-247 (2018).

9. Doctors, L.J. and Scrace, R.J. "The optimization of trimaran sidehull position for minimum resistance", FAST 2003, Ischia, Italy (2003).

10. Yanuar, Y., Gunawan, G., Talahatu, M.A., et al. "Resistance analysis of unsymmetrical trimaran model with outboard sidehulls configuration", J. Mar. Sci. Appl., 12(3), pp. 293-297 (2013).

11. Serani, A., Fasano, G., Liuzzi, G., et al. "Ship hydrodynamic optimization by local hybridization of deterministic derivative-free global algorithms", Applied Ocean Research., 59, pp. 115-128 (2016).

12. Daniali, O.A., Toghraie, D., and Eftekhari, S.A. "Thermo-hydraulic and economic optimization of Iranol refinery oil heat exchanger with Copper oxide nanoparticles using MOMBO", Phys. A Stat. Mech. its. Appl., 540, p. 123010 (2020).

13. Zhang, S., Zhang, B., Tezdogan, T., et al. "Computational fluid dynamics-based hull form optimization using approximation method", Engineering Applications of Computational Fluid Mechanics, 12(1), pp. 74-88 (2018).

14. Peri, D. and Campana, E.F. "High-fidelity models and multi-objective global optimization algorithms in simulation-based design", Journal of Ship Research, 49(3), pp. 159-175 (2005).

15. Mostafazadeh, A., Toghraie, D., Mashayekhi, R., et al. "Effect of radiation on laminar natural convection of nanofluid in a vertical channel with single- and twophase approaches", J. Therm Anal Calorim., 138(1), pp. 779-794 (2019).

16. Zhang, P., Zhu, D.X., and Leng, W.H. "Parametric approach to design of hull forms", J. Hydrodyn Ser. B., 20(6), pp. 804-810 (2008).

17. Pourfattah, F., Sabzpooshani, M., Toghraie, D., et al. "On the optimization of a vertical twisted tape arrangement in a channel subjected to MWCNT-water nanofluid by coupling numerical simulation and genetic algorithm", J. Therm. Anal. Calorim., 144(1), pp. 189-201 (2020).

18. Tahara, Y., Tohyama, S., and Katsui, T. "CFD-based multi-objective optimization method for ship design", Int. J. Numer. Methods Fluids, 52(5), pp. 499-527 (2006).

19. Chen, W.M. and Chen, X.P. "Application of CFD in ship hull lines optimization", J. SSSRI., 30(1), pp. 3032 (2007).
20. Brizzolara, S., Vernengo, G., Pasquinucci, C., et al. "Significance of parametric hull form definition on hydrodynamic performance optimization", In 6th International Conference on Computation Methods in Marine Engineering (Marine 2015), pp. 254-265 (2015).

21. Harries, S., Abt, C., and Hochkirch, K. "Modelling meets simulation-process integration to improve design", Sonderkolloquium zu Ehren der Professoren Hagen, Schluter und Thiel, Germany (2004).

22. Abt, C. "A new approach to integration of CAD and CFD for naval architects", In Sixth International Conference on Computer Applications and Information Technology in the Maritime Industries (COMPIT), Cortona (2007).

23. Harries, S. "Parametric design and hydrodynamic optimization of ship hull forms", PhD Thesis, Institut fur Schiffs-und Meerestechnik, Technische Universitat. Berlin, Germany (1998).

24. Han, S., Lee, Y.S., and Choi, Y.B. "Hydrodynamic hull form optimization using parametric models", $J$. Marine Sci. Technol., 17(1), pp. 1-17 (2012).

25. Vasudev, K.L., Sharma, R., and Bhattacharyya, S.K. "Multi-objective optimization design framework integrated with CFD for the design of AUVs", Methods Oceanography, 10, pp. 138-165 (2014).

26. Kim, K., Tilling, F., Bathfield, N., et al. "Hydrodynamic optimization of twin-skeg LNG ships by CFD and model testing", Int. J. Naval Archit. Ocean Eng., 6(2), pp. 392-405 (2014).

27. Kim, H. and Yang, C. "A new surface modification approach for CFD-based hull form optimization", Journal of Hydrodynamics, 22(5), pp. 520-525 (2010).

28. Huang, F. and Yang, C. "Hull form optimization of a cargo ship for reduced drag", J. Hydrodynamic, 28(2), pp. 173-183 (2016).

29. Diez, M., Campana, E.F., and Stern, F. "Stochastic optimization methods for ship resistance and operational efficiency via CFD", Struct Multidiscip Optim., 57(2), pp. 735-758 (2018).

30. Serani, A., Diez, M., Wackers, J., et al. "Stochastic shape optimization via design-space augmented dimensionality reduction and rans computations", AIA A Scitech 2019 Forum, January (2019).

31. Guo, J., Zhang, Y., Chen, Z., et al. "CFD-based multiobjective optimization of a waterjet-propelled trimaran", Ocean Eng., 195(November 2019), p. 106755 (2020).

32. Zakerdoost, H. and Ghassemi, H. "Hydrodynamic optimization of ship's hull-propeller system under multiple operating conditions using MOEA/D", J. Mar. Sci. Technol., 26(2), pp. 419-431 (2020).

33. Coppedè, A., Gaggero, S., Vernengo, G., et al. "Hydrodynamic shape optimization by high fidelity CFD solver and Gaussian process based response surface method", Appl Ocean Res., 90(May 2018), pp. 101841 (2019). 
34. Feng, Y., Moctar, O., and Schellin, T.E. "Hydrodynamic optimisation of a multi-purpose wind offshore supply vessel Hydrodynamic optimisation of a multipurpose wind offshore supply vessel", Sh. Technol. Res., $67(2)$, pp. 69-83 (2019).

35. Chen, X., Diez, M., Kandasamy, M., et al. "Highfidelity global optimization of shape design by dimensionality reduction, metamodels and deterministic particle swarm", Engineering Optimization, 47(4), pp. 473-494 (2015).

36. Campana, E.F., Peri, D., Tahara, Y., et al. "Numerical optimization methods for ship hydrodynamic design", In SNAME Annual Meeting (2009).

37. Akbari, K., Khedmati, M.R., and Seif, M.S. "Experimental study on heave and pitch motion characteristics of a wave-piercing trimaran", Transactions of Famena, 38(3), pp. 13-26 (2014).

38. Nazemian, A. and Ghadimi, P. "Shape optimisation of trimaran ship hull using CFD-based simulation and adjoint solver", Ships Offshore Struct., pp. 1-15 (Oct. 2020).

39. Ghadimi, P., Nazemian, A., and Sheikholeslami, M. "Numerical simulation of the slamming phenomenon of a wave-piercing trimaran in the presence of irregular waves under various seagoing modes", Proc. Inst. Mech. Eng. Part M. J. Eng. Marit. Environ. 233(4), pp. 1198-1211 (2019b).

40. User Guide. 2020. StarCCM+ version 2020.1. SIEMENS Simcenter.

41. ITTC Recommendations, "7.5-03-01-04 CFD, General CFD Verification", In ITTC - Recommended Procedures and Guidelines (2011b).

42. ITTC Recommendations, "Practical guidelines for ship CFD applications", In: Presented at the Proceedings of 26th ITTC, Hague (2011b).

43. Richardson, L. "The approximate arithmetical solution by finite differences of physical problems involving differential equations, with an application to the stresses in a masonry dam", Containing Papers of a Mathematical or Physical Character, IX., 210(459470), pp. 307-357 (1911).

44. ITTC Recommendations. "Uncertainty analysis in CFD, uncertainty assessment methodology and procedures", ITTC-Quality Manual, In Proceedings of the
International Towing Tank Conference, Venice, Italy, 8-14 September (2002).

45. ITTC Recommendations "Uncertainty analysis in CFD, verification and validation methodology and procedures", ITTC-Recommended Procedures and Guidelines, 7.5-03-01-01, In Proceedings of the International Towing Tank Conference, Wuxi, China, 18 September (2017).

46. Larsson, L. and Stern, F., An Assessment of the Gothenburg 2010 Workshop, Springer Pub. (2014).

47. Olivieri, A., Pistani, F., Avanzini, A., et al. "Towing tank experiments of resistance, sinkage and trim, boundary layer, wake, and free surface flow around a naval combatant insean 2340 model", Security, 421, pp. 1-42 (2001).

48. Wilcox, D.C., Turbulence Modeling for CFD, D.C.W. Industries, USA (2006).

\section{Biographies}

Amin Nazemian is a $\mathrm{PhD}$ candidate at the Department of Maritime engineering at the Amirkabir University of Technology in Tehran, Iran. He completed his both BSc and MSc from the Amirkabir University of Technology. His research interests include fluid-exposed geometry optimization, CFD, resistance and seakeeping analyses, and mathematical application in the hydrodynamics field and he co-authored four articles.

Parviz Ghadimi received his $\mathrm{PhD}$ in Mechanical Engineering in 1994 from Duke University, USA. He served one year as a Research Assistant Professor in Mechanical Engineering Department and six years as a Visiting Assistant Professor in Mathematics Department at Duke. He then joined the Dept. of Marine Technology at Amirkabir University of Technology, Iran, in fall 2005. He is currently a Full Professor of Hydromechanics at that Department. His main research interests include hydrodynamics, hydroacoustics, thermo-hydrodynamics, and CFD, and he has authored over two hundred scientific papers in these fields. 